TRANSACTIONS OF THE

AMERICAN MATHEMATICAL SOCIETY

Volume 350, Number 6, June 1998, Pages 2367-2391

S 0002-9947(98)01998-9

\title{
APPROXIMATION BY POLYNOMIALS WITH NONNEGATIVE COEFFICIENTS AND THE SPECTRAL THEORY OF POSITIVE OPERATORS
}

\author{
ROGER D. NUSSBAUM AND BERTRAM WALSH
}

Dedicated to Helmut H. Schaefer on the 70th anniversary of his birth

\begin{abstract}
For $\Sigma$ a compact subset of $\mathbf{C}$ symmetric with respect to conjugation and $f: \Sigma \rightarrow \mathbf{C}$ a continuous function, we obtain sharp conditions on $f$ and $\Sigma$ that insure that $f$ can be approximated uniformly on $\Sigma$ by polynomials with nonnegative coefficients. For $X$ a real Banach space, $K \subseteq X$ a closed but not necessarily normal cone with $\overline{K-K}=X$, and $A: X \rightarrow X$ a bounded linear operator with $A[K] \subseteq K$, we use these approximation theorems to investigate when the spectral radius $\mathrm{r}(A)$ of $A$ belongs to its spectrum $\sigma(A)$. A special case of our results is that if $X$ is a Hilbert space, $A$ is normal and the 1-dimensional Lebesgue measure of $\sigma\left(i\left(A-A^{*}\right)\right)$ is zero, then $\operatorname{r}(A) \in \sigma(A)$. However, we also give an example of a normal operator $A=-U-\alpha I$ (where $U$ is unitary and $\alpha>0$ ) for which $A[K] \subseteq K$ and $\operatorname{r}(A) \notin \sigma(A)$.
\end{abstract}

\section{INTRODUCTION}

If $X$ is a real Banach space and $K \subseteq X$ is a closed convex set, then $K$ is a closed cone (with vertex at zero) if (a) $\lambda K:=\{\lambda x: x \in K\} \subseteq K$ for all $\lambda \geq 0$ and (b) $K \cap(-K)=\{0\}$. If $K$ satisfies only condition (a), then $K$ is called a closed wedge. A closed cone or wedge $K$ will be called generating if $X=K-K$, and called total if $X=\overline{K-K}$. A closed cone $K$ induces a partial ordering of $X$ when $x \leq y$ is defined to mean $y-x \in K ; K$ is called normal if there exists a constant $M \geq 0$ such that $\|x\| \leq M\|y\|$ for all $x, y \in X$ with $0 \leq x \leq y$.

Suppose now that $X$ is a real Banach space, $K \subseteq X$ is a closed, total cone, and $A: X \rightarrow X$ is a positive bounded linear operator, i.e., one for which $A[K] \subseteq K$. Let $\sigma(A)$ denote the spectrum of the complexification $A_{\mathbf{C}}$ of $A$ and $\operatorname{r}(A)$ denote its spectral radius. This paper is concerned with the

Principal Question: Under what further conditions on $A$ is it true that $\operatorname{r}(A) \in$ $\sigma(A)$ ?

It is a classical result of Bonsall [4] and Schaefer [8] that if $K$ is normal and generating, then $\mathrm{r}(A) \in \sigma(A)$. However, Bonsall [4] gave an example of a closed,

Received by the editors December 26, 1995 and, in revised form, July 1, 1996.

1991 Mathematics Subject Classification. Primary 30C10, 47B15, 47B65.

Key words and phrases. Polynomial approximation with nonnegative coefficients, positive linear operators, spectral radius.

The first author was partially supported by NSF grant DMS 9401823.

(C)1998 American Mathematical Society 
generating cone $K$ in a Hilbert space $H$ and a positive bounded linear map $A$ : $H \rightarrow H$ for which $\mathrm{r}(A) \notin \sigma(A)$.

In an extremely interesting recent paper, Toland [12] was able to show that if $K$ is a closed, total cone in a real Hilbert space $H$ and $A$ is a bounded self-adjoint operator on $H$ with $A[K] \subseteq K$, then $\operatorname{r}(A) \in \sigma(A)$ must hold. Bonsall's counterexample suggests the delicacy of this result. The key step in Toland's argument is an approximation theorem which is independently interesting: for $a>1$, he shows that there exists a number $d$ with $1<d<a$ such that every continuous $f:[-a, 1] \rightarrow \mathbf{R}$ with support in $[-a,-d]$ can be approximated uniformly on $[-a, 1]$ by polynomials with nonnegative coefficients. In this paper, we shall generalize and sharpen this approximation theorem and use these results on approximation to attack the principal question.

The plan of this paper is the following. Section 1 below characterizes the duals of certain real Banach spaces of continuous complex-valued functions. Section 2 treats approximation questions. For $\Sigma$ a compact subset of $\mathbf{C}$ that is invariant under conjugation and $f: \Sigma \rightarrow \mathbf{C}$ a continuous function satisfying $f(\bar{z})=\overline{f(z)}$, we give sharp conditions under which $f$ can be approximated uniformly on $\Sigma$ by polynomials with nonnegative coefficients. These conditions imply in particular that the number $d$ in Toland's approximation theorem can be replaced by 1 . Section 3 applies these approximation results to the principal question. If $K$ is a closed, total cone in a real Hilbert space $H$ and $A$ is a bounded normal operator on $H$ such that $A[K] \subseteq K$ and $\sigma\left(i\left(A-A^{*}\right)\right)$ has 1-dimensional Lebesgue measure zero, then our results imply that $\mathrm{r}(A) \in \sigma(A)$. It is classical [4], [8] that if $K$ is a closed, total cone in a real Banach space $X$ and $A \in \mathcal{L}(X)$ is a positive operator whose resolvent function $R_{A}(\lambda)$ has a pole at some $\lambda_{1} \in \mathbf{C}$ with $\left|\lambda_{1}\right|=\operatorname{r}(A)$, then $\operatorname{r}(A) \in \sigma(A)$; a transparent demonstration of this fact, differing radically from previous proofs, follows from our approximation-theoretic approach.

These positive results might lead one to conjecture that $\mathrm{r}(A) \in \sigma(A)$ for every bounded, normal operator $A$ on a real Hilbert space $H$, such that $A$ takes a closed total cone $K \subseteq H$ into itself. In Section 4 , however, we show that this hope is unfounded: for the (unitary) shift operator $U$ on $\ell^{2}(\mathbf{Z})$ and any (fixed) $0<\alpha<1 / 3$, we construct a closed, total cone $K$ that is mapped into itself by $A=-U-\alpha I$, an operator whose spectrum is $\{\lambda-\alpha:|\lambda|=1\}$ and for which $\mathrm{r}(A) \in \sigma(A)$ therefore fails. However, for partial isometries mapping a closed, total cone into itself, the results of Section 3 imply that $1=\mathrm{r}(A) \in \sigma(A)$. Section 5 is essentially an appendix: in earlier sections of the paper it is necessary to refer to the process of complexifying real Banach spaces and to some of the details of spectral theory in that context, and this section presents those details in a convenient form.

Most of our notation is standard: $\mathbf{R}, \mathbf{C}$ and $\mathbf{D}$ denote the real numbers, the complex numbers and the closed unit $\operatorname{disc}\{\lambda \in \mathbf{C}:|\lambda| \leq 1\}$ respectively. The open unit disc is thus $\mathbf{D}^{\circ}$. The set of nonnegative real numbers is $\mathbf{R}^{+}$. The unit circle in the complex plane is $\mathbf{T}:=\partial \mathbf{D}$. Because the overbar conventionally denotes the conjugate $\bar{\lambda}$ of a complex number $\lambda$ but denotes the topological closure $\bar{V}$ of a set $V \subseteq \mathbf{C}$, we let $\operatorname{conj}(V):=\{\bar{\lambda}: \lambda \in V\}$ denote the set of complex numbers conjugate to those in a given set $V$. When we need a name for it, we denote the identity function on $\mathbf{C}$ by $z$. The (Fréchet-Montel) space of all holomorphic complex-valued functions on $\mathbf{D}^{\circ}$, equipped with the topology of uniform convergence on compacta, is denoted by $\mathcal{O}\left(\mathbf{D}^{\circ}\right)$. If $s$ is a point in a compact Hausdorff space $\Sigma$, then $\varepsilon_{s}$ denotes the "point mass at $s$ " measure on the Borel sets of $\Sigma$. 


\section{Functions Symmetric with Respect to an Involution}

This section is a discursive treatment of some facts about certain real Banach spaces of complex-valued continuous functions. The results are easy consequences of the (Riesz) representation theorem for the dual of $\mathcal{C}(\Sigma)$, where $\Sigma$ is a compact Hausdorff space. (No particular originality is claimed for this material, but the authors have found no convenient reference for it.) We give a general construction that we shall use in only one setting, of the most elementary kind; however, the generality in which we give it may make it easier to follow.

Let $\Sigma$ be a compact Hausdorff space and $U: \Sigma \rightarrow \Sigma$ a continuous involution (i.e., $U \circ U=\mathrm{id}_{\Sigma}$ ). Let $\mathrm{S}(\Sigma, U)=\mathrm{S}(\Sigma)=\mathrm{S}$ denote the set of continuous complexvalued functions on $\Sigma$ that satisfy $f(U(s))=\overline{f(s)}$ for all $s \in \Sigma$. S $(\Sigma)$ is obviously a closed real subalgebra of $\mathcal{C}(\Sigma, \mathbf{C})$ containing the real constants. Similarly, let $\mathcal{S}(\Sigma, U)=\mathcal{S}(\Sigma)=\mathcal{S}$ be the set of complex-valued regular Borel measures $\mu$ on $\Sigma$ satisfying $\mu(A)=\overline{\mu(U[A])}$ for all Borel sets $A \subseteq \Sigma$. $\mathcal{S}(\Sigma)$ is clearly a real subspace of the space $\mathcal{M}(\Sigma, \mathbf{C})$ of all complex-valued regular Borel measures, and it is closed in the total-variation norm.

We claim that the pairing

$$
\begin{aligned}
(\mathrm{S}, \mathcal{S}) & \rightarrow \mathbf{R}, \\
(f, \mu) & \rightarrow \int f d \mu,
\end{aligned}
$$

identifies $\mathcal{S}$ with the real norm dual $\mathcal{L}_{\mathbf{R}}(\mathrm{S}, \mathbf{R})$. First of all, the value of such an integral is evidently real, since for simple complex-valued Borel functions $f=$ $\sum \alpha_{j} \chi_{A_{j}}$ and $\mu \in \mathcal{S}$ we have

$$
\begin{aligned}
\int f d \mu & =\sum \alpha_{j} \mu\left(A_{j}\right)=\sum \alpha_{j} \overline{\mu\left(U\left[A_{j}\right]\right)} \\
\int \overline{f \circ U} d \mu & =\sum \overline{\alpha_{j}} \mu\left(U\left[A_{j}\right]\right)
\end{aligned}
$$

so if $f \equiv \overline{f \circ U}$, then the value of the integral is its own complex conjugate (and thus is real). Approximating functions $f \in \mathrm{S}$ by simple functions with the same symmetry property, we see that their integrals against measures in $\mathcal{S}$ are also real.

Next, it is obvious that the pairing is $\mathbf{R}$-bilinear. The pairing maps $\mathcal{S}$ isometrically into $\mathcal{L}_{\mathbf{R}}(\mathrm{S}, \mathbf{R})$, because if $\mu \in \mathcal{S}$ is given and $f \in \mathcal{C}(\Sigma, \mathbf{C})$ is chosen such that $\|f\|_{\infty} \leq 1$ and $\left|\int f d \mu\right| \geq|\mu|(\Sigma)-\epsilon$ - where without loss of generality we can have $\int f d \mu=\left|\int f d \mu\right|$ - we may set $g(s) \equiv(1 / 2)[f(s)+\overline{f(U(s))}]$, obtaining a function $g \in \mathrm{S}$ with $\|g\|_{\infty} \leq 1$ and

$$
\begin{aligned}
\int g d \mu & =\frac{1}{2}\left[\int f d \mu+\int \overline{(f \circ U)} d \mu\right]=\frac{1}{2}\left[\int f d \mu+\overline{\int f d \mu}\right] \\
& =\int f d \mu \geq|\mu|(\Sigma)-\epsilon .
\end{aligned}
$$

Finally, the map induced by the pairing is onto. To see this, we begin by observing that $\mathcal{C}(\Sigma, \mathbf{C})$ can be viewed as a complexification of $\mathrm{S}(\Sigma, U)$ (isomorphic to the one we construct externally below). Every element $f \in \mathcal{C}(\Sigma, \mathbf{C})$ can be written 
in the form $f=g+i \cdot h$ with $g, h \in \mathrm{S}(\Sigma, U)$ by writing

$$
f(s)=\frac{f(s)+\overline{f(U(s))}}{2}+i \cdot \frac{f(s)-\overline{f(U(s))}}{2 i} .
$$

The "components" are uniquely determined by $f$ because if $g(s)+i \cdot h(s) \equiv 0$, then conjugation of the function values gives $\overline{g(s)}-i \cdot \overline{h(s)} \equiv 0$ but composition with $U$ gives $g(U(s))+i \cdot h(U(s)) \equiv 0$, and the latter is equivalent to $\overline{g(s)}+i \cdot \overline{h(s)} \equiv 0$. Adding and subtracting those relations shows that $g(s) \equiv 0$ and $h(s) \equiv 0$ must hold. If $\Psi \in \mathrm{S}(\Sigma, U)_{\mathbf{R}}^{*}$ is given, it is straightforward to verify that

$$
\Phi: g+i \cdot h \rightarrow \Psi(g)+i \cdot \Psi(h) \quad(g, h \in \mathrm{S}(\Sigma, U))
$$

(well-)defines a $\mathbf{C}$-linear functional on $\mathcal{C}(\Sigma, \mathbf{C})$, so there is a uniquely determined regular complex-valued Borel measure $\mu \in \mathcal{M}(\Sigma)$ for which

$$
\Psi(g)+i \cdot \Psi(h)=\Phi(g+i \cdot h)=\int(g+i \cdot h) d \mu
$$

holds for all $g, h \in \mathrm{S}(\Sigma, U)$. In particular, therefore, $\int f d \mu \in \mathbf{R}$ holds for every $f \in$ $\mathrm{S}(\Sigma, U)$. If we define a Borel measure $\varphi$ by putting $\varphi(A)=\overline{\mu(U[A])}$ for Borel sets $A \subseteq \Sigma$, then approximation by simple functions shows us that $\int f d \varphi=\overline{\int \overline{f \circ U} d \mu}$, as it did above. It follows that $\int f d \varphi=\int f d \mu$ for all $f \in \mathrm{S}(\Sigma, U)$ and therefore for all $f \in \mathcal{C}(\Sigma)$, so by the uniqueness part of the Riesz representation theorem we have $\mu=\varphi$, i.e., $\mu \in \mathcal{S}(\Sigma, U)$, proving what we wished.

We shall call the functions and measures discussed above $U$-symmetric functions and $U$-symmetric measures respectively. The only case of this construction that will be of any interest below will be that in which $\Sigma$ is a subset of the complex plane that maps onto itself under complex conjugation and $U$ is $\lambda \rightarrow \bar{\lambda}$. In this case we shall simply call the functions and measures symmetric, or more explicitly conjugate-symmetric, and say that the set $\Sigma$ is symmetric with respect to $\mathbf{R}$. (Note that $\Sigma \cap \mathbf{R}=\emptyset$ is a possibility.)

\section{Uniform Approximation by Polynomials With Nonnegative Coefficients}

The setting for the material of this section is the following. $\Sigma$ is a non-empty compact conjugate-symmetric subset of $\mathbf{C}$. The number $\kappa(\Sigma)$ is defined by

$$
\kappa(\Sigma):= \begin{cases}\sup \left(\Sigma \cap \mathbf{R}^{+}\right) & \text {if } \Sigma \cap \mathbf{R}^{+} \neq \emptyset \\ 0 & \text { if } \Sigma \cap \mathbf{R}^{+}=\emptyset .\end{cases}
$$

The set $\Sigma$ will for the most part remain fixed for the remainder of this section, and $\kappa(\Sigma)$ may simply be abbreviated to $\kappa$. Define $\Sigma_{\kappa}$ by $\Sigma_{\kappa}:=\Sigma \cup\{\lambda:|\lambda| \leq \kappa(\Sigma)\}$. "Symmetry" in the sense of $\S 1$ above is conjugate-symmetry.

Recall that the Cauchy transform (cf. [3, p. 639]) of a complex-valued Borel measure $\mu \in \mathcal{M}(\mathbf{C})$ of compact support is defined by

$$
F(\lambda)=\int \frac{1}{\lambda-z} d \mu(z) .
$$

This function is defined on $\mathbf{C}$ except for a set of $\lambda$ of two-dimensional Lebesgue measure zero and is locally integrable, holomorphic off the support of $\mu$, and analytic 
at $\infty$ with the value zero there. If $\tau=\sup \{|\lambda|: \lambda \in \operatorname{supp}(\mu)\}$, then a routine computation with the geometric series shows that for $|\lambda|>\tau$,

$$
F(\lambda)=\sum_{n=1}^{\infty}\left[\int z^{n-1} d \mu(z)\right] \cdot \frac{1}{\lambda^{n}}
$$

That series is then the Taylor series of $F(\lambda)$ at $\infty$, and we have established the following simple fact.

Proposition 2.1. If $F(\lambda)$ is the Cauchy transform of a Borel measure of compact support in $\mathbf{C}$, then its Taylor series at $\infty$ is given by

$$
F(\lambda)=\sum_{n=1}^{\infty} \frac{a_{n}}{\lambda^{n}}, \quad \text { where } a_{n}=\int z^{n-1} d \mu(z), \quad n=0,1, \ldots .
$$

Most of the results given here follow from the following basic proposition. It is in turn a consequence of the fundamental theorem of A. Pringsheim, which we state in its simplest form for the reader's convenience (cf. [2, pp. 289-290] or [11, $\S \mathbf{7 . 2 1}$, pp. 214-215]).

Theorem [Pringsheim]. If all (except perhaps finitely many) of the coefficients of the power series $\sum_{n=0}^{\infty} c_{n} z^{n}$ satsify $c_{n} \geq 0$, and if the series has finite radius of convergence $\rho>0$, then $z=\rho$ is a singular point of the analytic function represented by the power series, and in fact the function element represented by the series cannot be continued analytically along $\mathbf{R}^{+}$beyond the point $\rho$.

Proposition 2.2. Let $\mu$ be a symmetric regular Borel measure on $\Sigma$ that satisfies

$$
\int z^{n} d \mu(z) \geq 0 \text { for } n=0,1, \ldots
$$

Suppose $V \subseteq\{\lambda \in \mathbf{C}:|\lambda|>\kappa(\Sigma)\}$ is an open set with the properties that $V \cap \Sigma$ has two-dimensional Lebesgue measure zero and that $V \backslash \Sigma$ is contained in the unbounded component of $\mathbf{C} \backslash \Sigma_{\kappa}$. Then the support of $\mu$ is contained in $\Sigma \backslash V$.

Proof. As we have just seen, the Taylor series at $\infty$ of $F(\lambda)$ is given by

$$
F(\lambda)=\sum_{n=0}^{\infty}\left[\int z^{n} d \mu(z)\right] \cdot \frac{1}{\lambda^{n+1}} .
$$

(Note that the functions $z^{n}$ are conjugate-symmetric on every conjugate-symmetric subset of $\mathbf{C}$.) The coefficients of the power series in $1 / \lambda$ of (2.06) are nonnegative, and therefore, by Pringsheim's theorem applied to the function $F(1 / \lambda)$, the power series converges in a "disc centered at $\infty$ " $\{\lambda:|\lambda|>\rho\}$ - where one has $\rho \geq 0$ (with equality possible), but in any event the function (element) defined by the power series of (2.06) has a singular point at $\rho \in \mathbf{R}^{+}$. The Cauchy transform $F(\lambda)$ is holomorphic in $\mathbf{C} \backslash \Sigma$, so the power series can have no singular point on $\mathbf{R}^{+}$to the right of $\kappa(\Sigma)$, and thus $\rho \leq \kappa$. By hypothesis, $V \backslash \Sigma$ is a subset of the unbounded component of $\mathbf{C} \backslash \Sigma_{\kappa}$, so by the identity theorem for holomorphic functions, the function defined by the power series agrees with the Cauchy transform of $\mu$ throughout $V \backslash \Sigma$. This is the relative complement in $\mathrm{V}$ of the set $V \cap \Sigma$, which we assumed was a null set for 2-dimensional Lebesgue measure; therefore, the sum of the power series and the Cauchy transform $F(\lambda)$ define the same distribution [10, Ch. I, $\S \S 2-3$; Tome I, pp. 25-26] in $V$. Since $\partial / \partial \bar{\lambda}$ applied to the sum of the power series gives zero throughout $V$, while [10, Ch. VI, formula (VI,10,20); Tome 
II, p. 71], $(1 / \pi) \cdot \partial F / \partial \bar{\lambda}$ defines the same distribution as $\mu$, we see that $\mu$ defines the zero distribution in $V$ and thus is the zero measure [10] there, as we set out to prove.

Remark. An alternative way of stating the hypothesis that $V \backslash \Sigma$ is contained in the unbounded component of $\mathbf{C} \backslash \Sigma_{\kappa}$ is the following: every point of $V \backslash \Sigma$ can be connected to a "disc with center $\infty$," in which the power series is known to converge to the Cauchy transform of $\mu$, by a path that does not pass through $\Sigma$ or through $\kappa \cdot \mathbf{D}$. Consequently, the effect of the hypothesis is to insure that the function to which the power series converges in $V \backslash \Sigma$ continues the Cauchy transform along such a path and must therefore equal the Cauchy transform in $V \backslash \Sigma$.

Theorem 2.3. Suppose $V \subseteq\{\lambda \in \mathbf{C}:|\lambda|>\kappa(\Sigma)\}$ is an open set with the properties that $V \cap \Sigma$ has two-dimensional Lebesgue measure zero and that $V \backslash \Sigma$ is contained in the unbounded component of $\mathbf{C} \backslash \Sigma_{\kappa}$. Then in order that a continuous symmetric complex-valued function $f \in \mathrm{S}(\Sigma)$ be the uniform limit on $\Sigma$ of polynomials with nonnegative coefficients, it is necessary and sufficient that $f \mid(\Sigma \backslash(V \cup \operatorname{conj}(V)))$ be the uniform limit on $\Sigma \backslash(V \cup \operatorname{conj}(V))$ of polynomials with nonnegative coefficients.

Proof. In order that $f$ be a uniform limit of such polynomials on all of $\Sigma$ it is clearly necessary that it be such a limit on every subset of $\Sigma$. Conversely, suppose a function $f \in \mathrm{S}(\Sigma)$ is not in the uniform closure in $\mathrm{S}(\Sigma)$ of the polynomials with nonnegative coefficients. Because the closure in $S(\Sigma)$ of the set of polynomials with nonnegative coefficients is convex, the Hahn-Banach theorem (in its "bipolar" or "separation" form [9, Ch. II, §9, Thm. 9.2, p. 65]) implies the existence of a linear functional $\Psi \in \mathrm{S}(\Sigma)^{*}$ for which $\Psi\left(z^{n}\right) \geq 0$ holds for $n=0,1, \ldots$ but $\Psi(f)<0$. By the characterization of $\mathrm{S}(\Sigma)^{*}$ given in $\S 1$ above, we know that there is a symmetric regular Borel measure $\mu$ for which $\Psi(g)=\int g d \mu$ for all $g \in \mathrm{S}(\Sigma)$. As the preceding proposition showed, however, the support of a symmetric measure $\mu$ for which $\int z^{n} d \mu \geq 0$ holds for all $n=0,1, \ldots$ must be contained in $\Sigma \backslash V$. Because $\mu$ is symmetric, $\Sigma \cap \operatorname{conj}(V)$ must also be $\mu$-null, and thus $\mu$ is supported in $\Sigma \backslash(V \cup \operatorname{conj}(V))$. Thus $\mu \in \mathcal{S}(\Sigma \backslash(V \cup \operatorname{conj}(V)))$ defines a linear functional on $\mathrm{S}(\Sigma \backslash(V \cup \operatorname{conj}(V)))$ that separates $f \mid(\Sigma \backslash(V \cup \operatorname{conj}(V)))$ from the monomials, and thus from the polynomials with nonnegative coefficients considered as functions on $\Sigma \backslash(V \cup \operatorname{conj}(V))$. But then $f \mid(\Sigma \backslash(V \cup \operatorname{conj}(V)))$ cannot be a uniform limit of such polynomials on $\Sigma \backslash(V \cup \operatorname{conj}(V))$.

Corollary 2.4. If $\Sigma$ and $V$ satisfy the hypotheses of the proposition, then any symmetric function $f$ for which $f \mid(\Sigma \backslash V)=0$ has the property that both it and its negative can be approximated uniformly on $\Sigma$ by polynomials with nonnegative coefficients.

In [12, Theorem 14], J. F. Toland gave an essentially constructive proof of the following proposition: If $[-a, 1] \subseteq \mathbf{R}$ is a closed real interval, with $a>1$ (so that the interval extends to the left of -1 ), then there exists a number $d$ with $1 \leq d<a$ such that any continuous real-valued function $f$ on $[-a, 1]$ that vanishes on $[-d, 1]$ can be approximated uniformly on $[-a, 1]$ by polynomials with nonnegative coefficients (and $-f$ can also be so approximated). In a personal conversation with one of the authors (R. D. N.), Toland raised the question of whether the corresponding statement would be true if $d$ were simply taken to be 1 . The affirmative answer is 
a consequence of the corollary just given; one need only observe that when $\Sigma \subseteq \mathbf{R}$, then (i) a function on $\Sigma$ is symmetric if and only if it is real-valued, and (ii) $\Sigma$ itself cannot have disconnected complement in C. Unfortunately, the proof we have given is totally nonconstructive.

Application 2.5. Let $[-a, 1] \subseteq \mathbf{R}$ be a closed real interval, with $a>1$ (so the interval extends to the left of -1$)$. Then any continuous real-valued function $f$ on $[-a, 1]$ that vanishes on $[-1,1]$ can be uniformly approximated on $[-a, 1]$ by polynomials with nonnegative coefficients, and so can $-f$.

Indeed, we need only take $\Sigma=[-a, 1]$; then $\kappa=1$ and we may take $V=\mathbf{C} \backslash \mathbf{D}$. Proposition 2.2 is easily seen to admit the following slight extension.

Corollary 2.6. Let $\mu$ be a symmetric regular Borel measure on $\Sigma$ with the property that there exists a constant $M \geq 0$ for which

$$
\int z^{n} d \mu(z) \geq-M \cdot \kappa^{n} \quad \text { for } n=0,1, \ldots .
$$

Suppose $V \subseteq\{\lambda \in \mathbf{C}:|\lambda|>\kappa(\Sigma)\}$ is an open set with the properties that $V \cap \Sigma$ has two-dimensional Lebesgue measure zero and that $V \backslash \Sigma$ is contained in the unbounded component of $\mathbf{C} \backslash \Sigma_{\kappa}$. Then the support of $\mu$ is contained in $\Sigma \backslash V$.

Proof. If $\kappa=0 \notin \Sigma$ the statement reduces to Proposition 2.2. Otherwise, the hypothesis on $\mu$ implies that

$$
\int z^{n} d\left(\mu+M \cdot \varepsilon_{\kappa}\right) \geq 0 \quad \text { for } n=0,1, \ldots
$$

and therefore, by virtue of that proposition, that the support of $\mu+M \cdot \varepsilon_{\kappa}$ is contained in $\Sigma \backslash V$. Since the support of that measure differs from that of $\mu$ by at most $\{\kappa\}$, which is contained in $\Sigma \backslash V$ in any event, the corollary follows.

This corollary leads to an instructive example. Let $\mu$ be normalized 1-dimensional Lebesgue measure on the unit circle $\mathbf{T}$. It is routine to verify that its Cauchy transform is given by

$$
\frac{1}{2 \pi} \int_{-\pi}^{\pi} \frac{d \theta}{\lambda-e^{i \theta}}= \begin{cases}1 / \lambda & \text { if }|\lambda|>1, \\ 0 & \text { if }|\lambda|<1,\end{cases}
$$

in $\mathbf{C} \backslash \mathbf{T}$. Thus the Cauchy transform of this measure has a discontinuity at each point of the unit circle. If $\mu_{\alpha}$ denotes the translate of this same measure to the circle of unit radius and center $-\alpha$ (note the sign!), where $0<\alpha<1$, then near $\infty$ the Cauchy transform of the translated measure is given by

$$
\frac{1}{2 \pi} \int_{-\pi}^{\pi} \frac{d \theta}{\lambda-\left(-\alpha+e^{i \theta}\right)}=\frac{1}{2 \pi} \int_{-\pi}^{\pi} \frac{d \theta}{(\lambda+\alpha)-e^{i \theta}}=\frac{1}{\lambda+\alpha}=\sum_{n=1}^{\infty} \frac{(-1)^{n-1} \alpha^{n-1}}{\lambda^{n}}
$$

(valid at least for $|\lambda|>1+\alpha$ ), and since the power series centered at $\infty$ of the Cauchy transform has the integrals $\left\{\int z^{n-1} d \mu_{\alpha}: n=1,2, \ldots\right\}$ as its coefficients, these are bounded below by -1 . On the other hand, if we multiply $\mu_{\alpha}$ by $\chi_{\mathbf{D}^{\circ}}$ i.e., consider only that part of $\mu_{\alpha}$ lying inside the open unit disc - then the fact that $\left|z^{n}\right| \leq 1$ in $\mathbf{D}$ gives us the (obvious) estimates

$$
\int z^{n} d\left(\chi_{\mathbf{D}^{\circ}} \cdot \mu_{\alpha}\right) \leq 1, \quad n=0,1, \ldots
$$


It follows that the measure $\nu:=\chi_{\left(\mathbf{C} \backslash \mathbf{D}^{\circ}\right)} \cdot \mu_{\alpha}+2 \cdot \varepsilon_{1}$, whose support is exactly the set $\Sigma=\{1\} \cup\{\lambda:|\lambda+\alpha|=1$ and $|\lambda| \geq 1\}$, satisfies $\int z^{n} d \nu \geq 0$ for all $n \geq 0$ - yet its support is not contained in the disc of radius $\kappa(\Sigma)=1$. What goes wrong, of course, is that if $\left|\lambda_{0}+\alpha\right|=1$ and $\left|\lambda_{0}\right|>1$, there does not exist an open neighborhood $V$ of $\lambda_{0}$ with $V \subseteq\{\lambda:|\lambda|>1\}$, such that $V \backslash \Sigma$ is contained in the unbounded component of $\mathbf{C} \backslash \Sigma_{1}$, even though $\mathbf{C} \backslash \Sigma$ and $\mathbf{C} \backslash \mathbf{D}$ are connected. The discontinuities of the Cauchy transform of $\mu_{\alpha}$ at the circle $\{\lambda:|\lambda+\alpha|=1\}$ make it impossible to remove the singularities of the Cauchy transform of $\nu$ on that circle.

This example furnishes us with a situation in which both the measure-theoretic lemma 2.2 and the approximation theorem 2.3 would fail if the topological hypothesis on $\Sigma$ were replaced by one of the weaker requirements that $V \backslash \Sigma$ be a subset of the unbounded component of the set $\mathbf{C} \backslash \Sigma$ or of $\mathbf{C} \backslash \kappa \cdot \mathbf{D}$ (rather than of $\mathbf{C} \backslash \Sigma_{\kappa}$ ). The measure $\nu$ is already a counterexample for the measure-theoretic lemma. For the approximation theorem, consider the function $f(z)=1-|z|^{2}$ on $\Sigma$. It is zero on the unit circle, but strictly negative on the rest of $\Sigma$. Therefore, $\int f(z) d \nu(z)<0$, but $\int z^{n} d \nu(z) \geq 0$ for $n=0,1, \ldots$. The measure $\nu$ is thus (an explicit example of) a measure separating $f(z)$ from the polynomials in $z$ with nonnegative coefficients, so it prevents $f(z)$ from being approximated uniformly on $\Sigma$ by those polynomials (note that $f(z)$ is itself a polynomial function in $x, y$, although it is not holomorphic).

While our principal applications of Theorem 2.3 to spectral-radius questions will be of the form suggested by Cor. 2.4 and App. 2.5 above, there are cases of the full theorem that may be of interest. The most simple of these is the following.

Proposition 2.7. Suppose that $\kappa=0$, that the two-dimensional Lebesgue measure of $\Sigma$ is zero and that $\Sigma$ has connected complement. Then when $0 \in \Sigma$ the uniform closure in $\mathrm{S}(\Sigma)$ of the polynomials with nonnegative coefficients consists of all functions $f \in \mathrm{S}(\Sigma)$ with $f(0) \geq 0$, and when $0 \notin \Sigma$ the uniform closure of the polynomials with nonnegative coefficients is all of $\mathrm{S}(\Sigma)$.

Proof. Both of these cases are cases of Theorem 2.3 with $V=\mathbf{C} \backslash\{0\}$, though in the case where $0 \notin \Sigma$ one is in the "vacuous case" of the theorem (the existence of the measure $\mu$ is untenable, because its support would be empty).

The first case of Prop. 2.7 puts the ingenious example of Bonsall [4, ex. (iii), p. $57 \mathrm{ff}$.] into a more general context. That example considers the (nonclosed proper) cone $V^{+}$of polynomial functions of the form $f(t)=\alpha_{0}-\alpha_{1} t+\cdots+(-1)^{n} \alpha_{n} t^{n}$ (all $\left.\alpha_{j} \geq 0\right)$ in $\mathcal{C}_{\mathbf{R}}[0,1]$, showing inter alia that this cone is far from normal and that its closure consists of all the continuous real functions on $[0,1]$ that are nonnegative at zero. Prop. 2.7 tells us that the uniform closure in $\mathcal{C}_{\mathbf{R}}[-1,0]$ of the polynomials with nonnegative coefficients consists of all the continuous real functions on that interval that are nonnegative at zero; the mapping $t \rightarrow-t$ of $[-1,0]$ to $[0,1]$ then produces the characterization of $\overline{V^{+}}$given in [4]. (The authors are indebted to the referee for this observation.)

The next most simple case of Theorem 2.3 is that in which the part of $\Sigma$ that lies outside the disc of radius $\kappa$ has two-dimensional Lebesgue measure zero and does not form "holes" with $\kappa \cdot \mathbf{D}$. For simplicity, let us take $\kappa=1$ and assume that $\Sigma \cap \mathbf{D}^{\circ}$ is dense in $\Sigma \cap \mathbf{D}$.

Proposition 2.8. Suppose that $\kappa=1$, that $\Sigma \cap \mathbf{D}^{\circ}$ is dense in $\Sigma \cap \mathbf{D}$, that the two-dimensional Lebesgue measure of $\Sigma \backslash \mathbf{D}$ is zero and that $\Sigma \cup \mathbf{D}$ has connected 
complement in the Riemann sphere. Then the uniform closure of the polynomials with nonnegative coefficients in $\mathrm{S}(\Sigma)$ consists of all functions $f \in \mathrm{S}(\Sigma)$ for which $f \mid(\Sigma \cap \mathbf{D})$ equals the restriction to $\Sigma \cap \mathbf{D}$ of a power series $\sum a_{j} z^{j}$ with each $a_{j} \geq 0$ and $\sum a_{j}<\infty$.

Proof. Theorem 2.3 clearly applies with $V=\mathbf{C} \backslash \mathbf{D}$, so proving the proposition reduces to showing that $f \mid(\Sigma \cap \mathbf{D})$ is a uniform limit on $\Sigma \cap \mathbf{D}$ of polynomials with nonnegative coefficients if and only if it is the restriction of the function defined by such a power series. It is clear that the restriction of such a power series is a uniform limit of polynomials with nonnegative coefficients on the whole disc $\mathbf{D}$. On the other hand, if $\left\{p_{n}(z)\right\}$ is a sequence of such polynomials that converges uniformly on $\Sigma \cap \mathbf{D}$, then (since each polynomial attains its maximum absolute value at $z=1)$ the $\left\{p_{n}(z)\right\}$ are uniformly bounded on $\mathbf{D}$. They therefore form a normal family in $\mathcal{O}\left(\mathbf{D}^{\circ}\right)$, and $\left\{p_{n}(z)\right\}$ thus contains a subsequence $\left\{p_{n_{k}}(z)\right\}$ that converges uniformly on compacta in $\mathbf{D}^{\circ}$ to a function $g(z)$ that is holomorphic in $\mathbf{D}^{\circ}$ and consequently has a power series representation $g(z)=\sum a_{j} z^{j}$ valid in $\mathbf{D}^{\circ}$. Since the power-series coefficients are continuous linear functionals on $\mathcal{O}\left(\mathbf{D}^{\circ}\right)$ and the coefficients of the $\left\{p_{n_{k}}\right\}$ were nonnegative, each $a_{j} \geq 0$ (and thus $g$ is symmetric on $\mathbf{D}^{\circ}$ ). The continuity of the power-series-coefficient functionals also implies that $\sum_{j=0}^{N} a_{j} \leq \lim \sup _{k \rightarrow \infty} p_{n_{k}}(1)$ for each fixed $N$, and thus that $\sum_{j=0}^{\infty} a_{j} \leq f(1)$. We may thus think of $g$ as having been (continuously) extended to all of $\mathbf{D}$, with its values $g(z)$ defined by the same power series. Since $g(z)=\lim _{k \rightarrow \infty} p_{n_{k}}(z)=f(z)$ holds at each $z \in\left(\Sigma \cap \mathbf{D}^{\circ}\right)$ and that set is dense in $\Sigma \cap \mathbf{D}$, we have $f \mid(\Sigma \cap \mathbf{D})=$ $g \mid(\Sigma \cap \mathbf{D})$, as desired.

One cannot assert in general that the power series $\sum_{j=0}^{\infty} a_{j} z^{j}$ of Proposition 2.8 is uniquely determined by the function $f \in \mathrm{S}(\Sigma)$, since there exist (nonzero) holomorphic functions on $\mathbf{D}$ that have absolutely convergent Taylor series with real coefficients but also have infinitely many zeros. To give a simple example, the function

$$
\varphi(z):= \begin{cases}\exp [(z+1) /(z-1)] & \text { if } z \neq 1, \\ 0 & \text { if } z=1,\end{cases}
$$

is bounded on $\mathbf{D}$, holomorphic on $\mathbf{C}$ except for its essential singularity at 1 , and takes the value $e^{-1}$ at the points $(1+i / n \pi)^{-1}, n \in \mathbf{Z} \backslash\{0\}$. Consequently, if we put

$$
\psi(z):=(z-1)^{5} \cdot\left(\varphi(z)-e^{-1}\right),
$$

then $\psi^{\prime \prime}(z)$ extends continuously to $\mathbf{D}$ (again with value zero at $z=1$ ), and therefore $\psi(z)$ has an absolutely convergent Taylor series (with real coefficients) $\psi(z)=$ $\sum_{j=0}^{\infty} b_{j} z^{j}$. If we now take $\Sigma=\left\{(1+i / n \pi)^{-1}: 0 \neq n \in \mathbf{Z}\right\} \cup\{1\}$, then the distinct power series

$$
\psi^{+}(z):=\sum_{j=0}^{\infty} b_{j}^{+} z^{j} \quad \text { and } \quad \psi^{-}(z):=\sum_{j=0}^{\infty} b_{j}^{-} z^{j}
$$

(neither of which is identically zero, because $\psi(1)=0$ ) represent the same function in $\mathrm{S}(\Sigma)$.

The characterization of the uniform closure of the polynomials with nonnegative coefficients in $\mathcal{C}_{\mathbf{R}}(\Sigma \cap \mathbf{D})$ as the functions given by power series with nonnegative 
coefficients whose sum is finite is, of course, only a slight generalization of the theorem of S. Bernstern (see [13, Ch. IV]) that characterizes absolutely monotone functions.

Probably the most interesting case of Proposition 2.8 is that in which $\Sigma=$ $[-a, 1] \subseteq \mathbf{R}$ for some $a>1$ (so $\mathrm{S}(\Sigma)$ is simply $\mathcal{C}_{\mathbf{R}}[-a, 1]$ and $\left.\mathcal{S}(\Sigma)=\mathcal{M}_{\mathbf{R}}[-a, 1]\right)$. In this case various alternative choices can replace the monomials $\left\{z^{n}: n=0,1, \ldots\right\}$ : if for each $n=0,1, \ldots$ we are given a polynomial $P_{n}(x)$ of degree $n$ with positive leading coefficient, and if the $\left\{P_{k}(x): k=0,1, \ldots\right\}$ have the property that for each $n=0,1, \ldots$ there are nonnegative coefficients $\left\{\alpha_{n, k}: k=0, \ldots, n\right\}$ with $x^{n}=\sum_{k=0}^{n} \alpha_{n, k} P_{k}(x)-i . e .$, the wedge in $\mathcal{C}_{\mathbf{R}}[-a, 1]$ generated by the $\left\{P_{k}(x)\right.$ : $k=0,1, \ldots\}$ contains the wedge generated by the monomials - then the functions that can be uniformly approximated by polynomials with nonnegative coefficients can also be approximated by linear combinations $\sum_{k=0}^{n} \alpha_{k} P_{k}(x)$ with nonnegative coefficients. The usual Bernoulli polynomials defined by

$$
\frac{t e^{t x}}{e^{t}-1}=\sum_{n=0}^{\infty} B_{n}(x) \frac{t^{n}}{n !}, \quad \text { or } \quad e^{t x}=\frac{e^{t}-1}{t} \sum_{n=0}^{\infty} B_{n}(x) \frac{t^{n}}{n !}
$$

are an example, since the second formula shows that each $x^{n}$ is a linear combination of $B_{0}(x), \ldots, B_{n}(x)$ with positive coefficients. Similarly the Bernoulli polynomials adjusted to fit $[-1,1]$, i.e., $\left\{2^{n} \cdot B_{n}((x+1) / 2): n=0,1, \ldots\right\}$, are generated by

$$
\frac{t}{\sinh t} e^{t x}=\frac{2 t e^{t(x+1)}}{e^{2 t}-1}=\sum_{n=0}^{\infty} B_{n}\left(\frac{x+1}{2}\right) \frac{(2 t)^{n}}{n !} ;
$$

since this relation is equivalent to

$$
\sum_{n=0}^{\infty} x^{n} \frac{t^{n}}{n !}=\frac{\sinh t}{t} \cdot \sum_{n=0}^{\infty} 2^{n} \cdot B_{n}\left(\frac{x+1}{2}\right) \frac{t^{n}}{n !},
$$

and the power-series coefficients of $\sinh t$ are nonnegative, the $\left\{2^{n} \cdot B_{n}((x+1) / 2)\right\}$ have this property. The extension to other suitable Appell polynomials is obvious. Another class of examples is furnished by the orthogonal polynomials belonging to a symmetric weight on $[-1,1]$. It is well known (see, e.g., [1, p. 44], with a different notational convention) that such (monic) polynomials satisfy a recurrence relation of the form

$$
P_{n}(x)=x \cdot P_{n-1}(x)-\lambda_{n} P_{n-2}(x),
$$

where $\lambda_{n}>0$ for $n \geq 1$, with $P_{0}(x) \equiv 1$ and $P_{1}(x) \equiv x$. It follows from such a recurrence that the wedge generated by the $\left\{P_{n}(x): n=0,1, \ldots\right\}$ in $\mathcal{C}_{\mathbf{R}}[-a, 1]$ contains the monomials: the presence of 1 and $x$ is assured, and if for $k \geq 1$

$$
x^{k}=\beta_{k, 0}+\beta_{k, 1} x+\sum_{n=2}^{k} \beta_{k, n} P_{n}(x),
$$

then

$$
\begin{aligned}
x^{k+1} & =\beta_{k, 0} x+\sum_{n=1}^{k} \beta_{k, n} x \cdot P_{n}(x) \\
& =\beta_{k, 0} P_{1}(x)+\sum_{n=1}^{k} \beta_{k, n}\left[P_{n+1}(x)+\lambda_{n+1} P_{n-1}(x)\right] .
\end{aligned}
$$


For some choices of $\left\{P_{n}(x)\right\}$, e.g., Gegenbauer polynomials, it is possible to compute these coefficients explicitly; see $[5, \S 7.1]$. In all cases, a version of Theorem 2.3 is available for approximations by linear combinations with nonnegative coefficients of polynomials of these kinds.

Proposition 2.9. Let $\left\{P_{n}(x): n=0,1, \ldots\right\}$ be monic polynomials with $\operatorname{deg} P_{n}=$ $n$, and suppose the closed wedge they generate in $\mathcal{C}_{\mathbf{R}}[-a, 1]$ contains all the monomials $\left\{x^{n}: n=0,1, \ldots\right\}$. Let $a>1$. Then in order that $f \in \mathcal{C}_{\mathbf{R}}[-a, 1]$ be the uniform limit on $[-a, 1]$ of polynomials of the form $\sum_{n=0}^{N} \alpha_{n} P_{n}(x)$ with coefficients $\alpha_{n} \geq 0$, it is necessary and sufficient that $f \mid[-1,1]$ be the uniform limit on $[-1,1]$ of such polynomials.

Proof. The necessity of the condition is clear. For sufficiency, suppose $f \in \mathcal{C}_{\mathbf{R}}[-a, 1]$ satisfied the condition but were not in the closed wedge in $\mathcal{C}_{\mathbf{R}}[-a, 1]$ generated by such polynomials. Then the Hahn-Banach theorem would (again) furnish a measure $\mu \in \mathcal{M}_{\mathbf{R}}[-a, 1]$ with $\int f d \mu<0$ but $\int g d \mu \geq 0$ for every $g \in \mathcal{C}_{\mathbf{R}}[-a, 1]$ belonging to that closed wedge, which would entail $\int x^{n} d \mu \geq 0$ for $n=0,1, \ldots$. Applying Proposition 2.2 (with $V=\mathbf{C} \backslash \mathbf{D}$ ), we would see that $\operatorname{supp}(\mu) \subseteq[-1,1]$, so $\mu \in \mathcal{M}_{\mathbf{R}}[-1,1]$ would separate $f \mid[-1,1]$ from the wedge in $\mathcal{C}_{\mathbf{R}}[-1,1]$ generated by the $\left\{P_{n}(x)\right\}$, contrary to hypothesis.

In certain cases it is possible to give a strong characterization of the closed wedges in $\mathcal{C}_{\mathbf{R}}[-1,1]$ generated by a family of orthogonal polynomials. The wedges generated by Čebyšev polynomials, or more generally by Gegenbauer polynomials, are particularly easy to characterize. Recall that these are the polynomials with generating functions

$$
\frac{1-r x}{1-2 r x+r^{2}}=\sum_{n=0}^{\infty} T_{n}(x) r^{n} \quad \text { and } \quad \frac{1}{\left(1-2 r x+r^{2}\right)^{\lambda}}=\sum_{n=0}^{\infty} C_{n}^{\lambda}(x) r^{n}
$$

respectively.

Proposition 2.10. Let $\left\{P_{n}(x): n=0,1, \ldots\right\}$ be the Čebyšev polynomials $\left\{T_{n}(x)\right\}$ or the Gegenbauer polynomials $\left\{C_{n}^{\lambda}(x)\right\}, \lambda>0$. In order that a function $f \in$ $\mathcal{C}_{\mathbf{R}}[-1,1]$ be a uniform limit on $[-1,1]$ of polynomials $p(x)=\sum_{k=0}^{n} \alpha_{k} P_{k}(x)$ with all $\alpha_{k} \geq 0$, it is necessary and sufficient that $f$ have the form $f(x)=\sum_{k=0}^{\infty} \alpha_{k} P_{k}(x)$, where $\sum_{k=0}^{\infty} \alpha_{k} P_{k}(1)<\infty$ with all $\alpha_{k} \geq 0$; the series converges uniformly and absolutely on $[-1,1]$.

Proof. For either of these $\left\{P_{n}(x)\right\}$ one may replace $x$ by $\cos \theta$ in the generating function, factorize $\left(1-2 r \cos \theta+r^{2}\right)^{-\lambda}=\left(1-r e^{i \theta}\right)^{-\lambda}\left(1-r e^{-i \theta}\right)^{-\lambda}$, expand the two factors in binomial series and multiply the series to display $P_{n}(\cos \theta)$ as a linear combination $\sum_{k=0}^{n} \gamma_{k, n} \cos k \theta$ in which all coefficients are nonnegative. (Indeed, the Čebyšev polynomials are [more fundamentally] characterized by the identity $T_{n}(\cos \theta) \equiv \cos n \theta$.) It follows that these $\left\{P_{n}(x)\right\}$ attain their (positive) absolute maxima on $[-1,1]$ at $x=1$. Consequently, if $f$ does have the form $f(x)=\sum_{k=0}^{\infty} \alpha_{k} P_{k}(x)$, where $\sum_{k=0}^{n} \alpha_{k} P_{k}(1)<\infty$ with all $\alpha_{k} \geq 0$, then the series converges uniformly and absolutely on $[-1,1]$ to an element of $\mathcal{C}_{\mathbf{R}}[-1,1]$ which clearly belongs to the uniformly closed wedge generated by the $\left\{P_{n}(x): n=0,1, \ldots\right\}$. Conversely, if there exists a sequence of polynomials $\left\{p_{k}(x)\right\}$ converging uniformly on $[-1,1]$ to $f \in \mathcal{C}_{\mathbf{R}}[-1,1]$, with each having the form $p_{k}(x)=\sum_{n=0}^{\operatorname{deg} p_{k}} \beta_{k, n} P_{n}(x)$ with all $\beta_{k, n} \geq 0$, then the Fourier coefficients of 
the $\left\{p_{k}(x)\right\}$ with respect to the system $\left\{P_{n}(x)\right\}$ converge to those of $f$. If the coefficients of $f$ are denoted by $\left\{\alpha_{n}: n=0,1, \ldots\right\}$, we thus have $\alpha_{n} \geq 0$ for each $n$. For each fixed $N$ the relation $\sum_{n=0}^{N} \alpha_{n} P_{n}(1) \leq \lim \sup _{k \rightarrow \infty} p_{k}(1)$ holds, and therefore $\sum_{n=0}^{\infty} \alpha_{n} P_{n}(1)<\infty$; since this series converges uniformly but also converges to $f$ in $L_{w}^{2}[-1,1]$ for the appropriate weight function $w(x)$, we have $f(x)=\sum_{n=0}^{\infty} \alpha_{n} P_{n}(x)$, with the series converging absolutely and uniformly on $[-1,1]$.

\section{Sufficient Conditions for the Spectral Radius to Belong to the Spectrum}

This section is devoted to application of the approximation-theoretic results of $\S 2$ to the question: If $X$ is a real Banach space, $K \subseteq X$ is a closed cone which may not be normal or generating but for which $X=\bar{K}-K$ (i.e., a total cone), and $A: X \rightarrow X$ is a linear operator for which $A[K] \subseteq K$, must the spectral radius $\mathrm{r}(A)$ belong to $\sigma(A)$ ? We extend the results of [12] to a significant extent, and give a new proof of the theorem ([4], [9, App. §2, Thm. 2.4, pp. 264-265]) that if the resolvent of such an operator has a pole on the "spectral circle" $\{\lambda:|\lambda|=\operatorname{r}(A)\}$, then $\mathrm{r}(A) \in \sigma(A)$.

In what follows, references to $X$ and $K$ will be assumed to fit into the pattern just described, except that when the space is a Hilbert space we shall call it $H$. The crucial property of a total cone $K$ in all cases is that if $B \in \mathcal{L}(X)$ is an operator for which $B[K]=\{0\}$, then $B=0$. It is straightforward to verify that if $T: H \rightarrow H$ commutes with $T^{*}$ on $H$, then $T_{\mathbf{C}}$ is a normal operator on the complex Hilbert space $H_{\mathbf{C}}$, and that if $V$ is a partial isometry $\left(V^{*} V=I\right)$ on $H$, then its complexification is a partial isometry on $H_{\mathbf{C}}$ which is unitary if and only if $V V^{*}=I$ on the real Hilbert space $H$.

Although we employ App. 2.5 as a convenience in proving the following proposition, it could as well have been established using the approximation theorem of Toland [12, Theorem 14]. The proof requires a spectral mapping theorem for the $C^{k}$ operational calculi of S. Kantorovitz; while this theorem could be deduced from [6, Lemma 2.2], we give a short self-contained proof as our Lemma 3.2. As an example of an application of the proposition, we observe that its hypotheses are satisfied when $A$ is an operator of the form $B+N$ on a Hilbert space, where $B$ is self-adjoint, $N$ is nilpotent, and $B N=N B$.

Proposition 3.1. Let $A \in \mathcal{L}_{\mathbf{R}}(X)$ have the property that, for some integer $k$, $\|\cos (t A)\|=O\left(|t|^{k}\right)$ and $\|\sin (t A)\|=O\left(|t|^{k}\right)$ as $|t| \rightarrow \infty$. Then $\sigma(A) \subseteq \mathbf{R}$, and if $A[K] \subseteq K$ then $\mathrm{r}(A) \in \sigma(A)$.

Proof. The growth condition on $\cos (t A)$ and $\sin (t A)$ is equivalent to the growth condition $\left\|\exp \left(i t A_{\mathbf{C}}\right)\right\|=O\left(|t|^{k}\right)$ on $A_{\mathbf{C}}$, so by [6, Theorem 2.14 and Lemma 2.11] we know that $\sigma(A) \subseteq \mathbf{R}$ and that $A_{\mathbf{C}}$ admits a $C^{k+2}$ operational calculus, i.e., there is a continuous homomorphism $f \rightarrow f(A)$ of $C_{0}^{k+2}(\mathbf{R})$ into $\mathcal{L}\left(X_{\mathbf{C}}\right)$ given by $f(A)=\int \hat{f}(t) \exp (i t A) d t$ (where $\hat{f}(t)=(1 / 2 \pi) \int f(x) \exp (-i t x) d x$, the usual Fourier transform of $f$ [up to normalization]) which is supported by $\sigma(A)$ and satisfies a boundedness condition of the form

$$
\begin{aligned}
\|f(A)\| \leq & \text { Const. } \cdot(b-a) \\
& \cdot\left[\max \{|f(x)|: a \leq x \leq b\}+\max \left\{\left|f^{(k+2)}(x)\right|: a \leq x \leq b\right\}\right]
\end{aligned}
$$

for each interval $[a, b]$ containing $\sigma(A)$ in its interior, and which extends to a continuous homomorphism of $C^{k+2}(\mathbf{R}) \rightarrow \mathcal{L}\left(X_{\mathbf{C}}\right)$ satisfying $1(A)=1$ and $z(A)=A$ (and 
also the norm-estimate condition (3.01)). By straightforward verification that the relations between the complex Fourier transform and the real cosine and sine Fourier transforms carry over to the complexified-operator situation, one shows that if $f$ is a real-valued $C^{k+2}$-function of compact support and $g(t)=(1 / 2 \pi) \int f(x) \cos (x t) d x$ and $h(t)=(1 / 2 \pi) \int f(x) \sin (x t) d x$ are its cosine and sine Fourier transforms respectively, then

$$
\int_{-\infty}^{\infty} \hat{f}(t) e^{i t A_{\mathbf{C}}} d t=\int_{-\infty}^{\infty} g(t) \cos \left(t A_{\mathbf{C}}\right) d t+\int_{-\infty}^{\infty} h(t) \sin \left(t A_{\mathbf{C}}\right) d t
$$

which is a real operator, namely, the complexification of

$$
\int g(t) \cos (t A) d t+\int h(t) \sin (t A) d t \in \mathcal{L}_{\mathbf{R}}(X)
$$

Suppose now that $A$ satisfies the hypotheses of the proposition, in particular that $A[K] \subseteq K$, but that $\mathrm{r}(A) \notin \sigma(A)$. Without loss of generality, multiplying $A$ by a suitable positive constant if necessary, we can assume that $\sigma(A) \subseteq[-d, 1)$, where $d=\mathrm{r}(A)>1$. Let $0 \leq f \in C_{0}^{\infty}(\mathbf{R})$ be a function with $f(-d)>0, f[[-1,1]]=\{0\}$, and support contained in $[-2 d, 1]$. Let $\left\{\varphi_{n}(x)\right\}$ be a sequence of polynomials with nonnegative coefficients converging uniformly to $f^{(k+2)}$ on $[-2 d, 1]$; such a sequence exists in virtue of App. 2.5 above. If $L$ denotes the "integration-from-zero" operator on $C^{\infty}(\mathbf{R})$, so $[L g](x)=\int_{0}^{x} g(t) d t$, then, since $f$ and all its derivatives vanish at zero, a simple uniform-convergence argument shows that the sequence $\left\{p_{n}(x):=\right.$ $\left.\left[L^{k+2} \varphi_{n}\right](x)\right\}$ - whose elements are polynomials with nonnegative coefficients converges to $f$ uniformly on a neighborhood of $\sigma(A) \subseteq \mathbf{R}$, and that the same is true of all derivatives of order $\leq(k+2)$. It follows from the norm estimate (3.01) that the sequence $\left\{p_{n}(A)\right\}$ converges to $f(A)$ in the norm topology of $\mathcal{L}_{\mathbf{R}}(X)$. Since $p_{n}(A)[K] \subseteq K$ for each $n$, and $K$ is closed, we have $f(A)[K] \subseteq K$; but since exactly the same approximation procedure could have been applied to $-f$, we have $f(A)[K] \subseteq-K$ and therefore $f(A)[K]=\{0\}$, whence $f(A)=0$ because $K$ is proper and $X=\overline{K-K}$. Because $0<f(-d) \in \sigma(f(A))$ by the spectral mapping theorem (Lemma 3.2 below), this is a contradiction; the proposition is therefore established.

Lemma 3.2. If $A \in \mathcal{L}_{\mathbf{R}}(X)$ satisfies the hypothesis of polynomial growth of $\cos (t A)$ and $\sin (t A)$ of Prop. 3.1 above (and thus admits a $C^{k+2}(\mathbf{R})$ operational calculus), then for any $f \in C^{k+2}(\mathbf{R})$ the relation $\sigma(f(A))=f[\sigma(A)]$ holds.

Proof. It follows from the characterization of the support and the estimate (3.01), or from [6], Lemma 2.5], that $\sigma(f(A)) \subseteq f[\sigma(A)]$. To see the reverse inclusion, let $\mathcal{A} \subseteq$ $\mathcal{L}_{\mathbf{C}}\left(X_{\mathbf{C}}\right)$ be the norm-closure of the polynomials in $A_{\mathbf{C}} ; \mathcal{A}$ is a commutative Banach algebra containing $I$ and is the same as the norm-closure of the rational functions of $A$ with poles off $\sigma(A)$. [This is true by a familiar argument: if $\Phi \in\left[\mathcal{L}_{\mathbf{C}}\left(X_{\mathbf{C}}\right)\right]^{*}$ annihilates all polynomials in $A$ then the resolvent series shows that it annihilates $(\lambda I-A)^{-1}$ for large $|\lambda|$, but since $\mathbf{C} \backslash \sigma(A)$ is connected, $\Phi$ annihilates all $(\lambda I-A)^{-1}$ for $\lambda \in \rho(A)$; since $\Phi$ was arbitrary, all the operators $\left\{(\lambda I-A)^{-1}: \lambda \in \rho(A)\right\}$ therefore belong to $\mathcal{A}$, which is an algebra.] In particular, spectra of elements of $\mathcal{A}$ relative to $\mathcal{A}$ are therefore equal to their spectra relative to $\mathcal{L}_{\mathbf{C}}\left(X_{\mathbf{C}}\right)$. It is evident from the definition of $f(A)$ for $f \in C^{k+2}(\mathbf{R})$, or from $(3.02)$ above, that $f(A) \in \mathcal{A}$. For any $f \in C^{k+2}(\mathbf{R})$ we can find a sequence $\left\{p_{n}\right\}$ of polynomials converging to $f$ in the topology of that space, i.e., uniformly on compacta in $\mathbf{R}$ together with their derivatives up to order $(k+2)$. The norm inequality $(3.01)$ shows that the $\left\{p_{n}(A)\right\}$ 
then converge to $f(A)$, so their Gelfand transforms (denoted by ${ }^{\wedge}$ ) on the maximal ideal space $\widehat{\mathcal{A}}$ of $\mathcal{A}$ converge uniformly to $\widehat{f(A)}$. If $\lambda \in \sigma(A)$ then there is a point $\xi \in \widehat{\mathcal{A}}$ with $\lambda=\widehat{A}(\xi)$, but then $\widehat{f(A)}(\xi)=\lim _{n \rightarrow \infty} \widehat{p_{n}(A)}(\xi)=\lim _{n \rightarrow \infty} p_{n}(\widehat{A}(\xi))=$ $\lim _{n \rightarrow \infty} p_{n}(\lambda)=f(\lambda)$, so $f(\lambda) \in \sigma(f(A))=\widehat{f(A)}[\widehat{\mathcal{A}}]$. Thus the spectral mapping theorem holds for such an operational calculus, i.e., $\sigma(f(A))=f[\sigma(A)]$.

A sufficient condition of a different type, involving the structure of the spectrum, holds for normal operators on a real Hilbert space $H$ ordered by a closed cone $K$ for which $H=\overline{K-K}$.

Proposition 3.3. Let $T$ be a normal operator on a real Hilbert space ordered by a closed, proper, total cone $K$ for which $T[K] \subseteq K$. Suppose that the spectrum of the imaginary part of $T_{\mathbf{C}}$ has one-dimensional Lebesgue measure zero. Then $\mathrm{r}(T) \in \sigma(T)$.

Proof. Let $T_{\mathbf{C}}=A+i B$ be the decomposition of $T_{\mathbf{C}}$ into its real and imaginary parts. Let $\alpha=\min \sigma(A)$ and $a=\max \sigma(A)$, and set $\Sigma_{*}:=[\alpha, a] \times \sigma(B)$ (as a subset of $\mathbf{C}$ considered as $\mathbf{R}^{2}$ ); then $\Sigma_{*}$ has two-dimensional Lebesgue measure zero. Since $A$ and $B$ commute, the Gelfand theory of commutative Banach algebras shows that $\sigma\left(T_{\mathbf{C}}\right) \subseteq \sigma(A)+i \cdot \sigma(B)$, and of course the latter set is a subset of $\Sigma_{*}$. Let $\Sigma:=\sigma\left(T_{\mathbf{C}}\right)$, which as the spectrum of a complexified operator is symmetric. If $\kappa=\sup \left(\Sigma \cap \mathbf{R}^{+}\right)$and $V=\{\lambda \in \mathbf{C}:|\lambda|>\kappa\}$, then $V$ satisfies the hypotheses of Theorem 2.3. Indeed, the dense open set $V \backslash \Sigma_{*}$ is connected - it contains with each of its points the horizontal line whose ordinate is the ordinate of the point and a fortiori $V \backslash \Sigma$ is connected; the same argument, using "half horizontal lines" where necessary, shows that $V \backslash \Sigma_{\kappa}$ (which is dense in $\mathbf{C} \backslash \Sigma_{\kappa}$ ) is connected. If it were true that $\kappa<\mathrm{r}\left(T_{\mathbf{C}}\right)$, then we could find $f \in \mathrm{S}(\Sigma)$, e.g., $f(\lambda):=\operatorname{dist}(\lambda, \kappa \cdot \mathbf{D})$, which vanished identically on $\kappa \cdot \mathbf{D}$ but for which $f\left(\lambda_{1}\right)>0$ for some $\lambda_{1} \in \sigma\left(T_{\mathbf{C}}\right)$ with $\left|\lambda_{1}\right|=\mathrm{r}\left(T_{\mathbf{C}}\right)$. There would then exist a sequence $\left\{p_{n}(x)\right\}$ of polynomials with nonnegative coefficients converging uniformly to $f$ on $\sigma\left(T_{\mathbf{C}}\right)$, so $\left\{\left(p_{n}(T)\right)_{\mathbf{C}}\right\}$ would converge to $f\left(T_{\mathbf{C}}\right)$ in operator norm; $f\left(T_{\mathbf{C}}\right)$ would thus be a real operator and map $K$ into itself. But again, the same would hold true of $-f\left(T_{\mathbf{C}}\right)$ (with a different approximating sequence), so we would have $f\left(T_{\mathbf{C}}\right)=0$, contrary to the spectral mapping theorem, which implies that $\sigma\left(f\left(T_{\mathbf{C}}\right)\right)$ contains $f\left(\lambda_{1}\right)>0$.

The following proposition can be proved by essentially the same method.

Proposition 3.4. Let $H$ be a real Hilbert space ordered by a closed, total cone $K$. Suppose $U, A \in \mathcal{L}(H)$ are commuting operators with $U^{*}=U^{-1}$ and $A=A^{*} \geq 0$, and that $T=U A$ has the property $T[K] \subseteq K$. If one of $\sigma(U)$ and $\sigma(A)$ has onedimensional Lebesgue measure zero, then $\mathrm{r}(T) \in \sigma(T)$.

Proof sketch. Under either of the measure-theoretic hypotheses, $\sigma(T) \subseteq \Sigma_{*}:=$ $\sigma(U) \cdot \sigma(A)$, and the latter set has two-dimensional Lebesgue measure zero. If $\kappa=\sup \left(\sigma\left(T_{\mathbf{C}}\right) \cap \mathbf{R}^{+}\right)$and $V=\{\lambda \in \mathbf{C}:|\lambda|>\kappa\}$, then $V$ satisfies the hypotheses of Theorem 2.3 with $\Sigma=\sigma\left(T_{\mathbf{C}}\right)$, though the reasons are different in the two cases. Again $\Sigma$, as the spectrum of a complexified operator, is symmetric. If it was assumed that $\sigma(A)$ had one-dimensional Lebesgue measure zero, then any point in $V \backslash \Sigma$ is the center of a disc in $V \backslash \Sigma$ that meets $V \backslash \Sigma_{*}$, and any point in the latter set can be joined to the open ray $(\kappa, \infty) \subseteq \mathbf{R}^{+}$by an arc of a circle lying entirely in $V \backslash\left(\Sigma_{*} \cup \kappa \cdot \mathbf{D}\right)$; thus $V \backslash \Sigma$ is (arc-)connected to the unbounded 
component of $\mathbf{C} \backslash \Sigma_{\kappa}$. If it was assumed that $\sigma(U)$ had one-dimensional Lebesgue measure zero, then any point in $V \backslash \Sigma$ is the center of a disc in $V \backslash \Sigma$ that meets $\left\{\rho e^{i \theta}: \kappa<\rho \in \mathbf{R}^{+}, e^{i \theta} \notin \sigma(U)\right\}$, and all points in the latter set are obviously (arc-) connected to the unbounded component of $\mathbf{C} \backslash \Sigma_{\kappa}$. If it were true that $\kappa<\mathrm{r}\left(T_{\mathbf{C}}\right)$, a consequence contrary to the spectral mapping theorem would now follow as in the proof of Prop. 3.3.

Prop. 3.4 includes as a special case operators with $U^{*}=U^{-1}$ (unitary transformations, or perhaps "orthogonal transformations" since the scalar field is $\mathbf{R}$ ). In fact, it includes the case of partial isometries, i.e., operators $V \in \mathcal{L}(H)$ for which $V^{*} V=I$, but for a reason that has nothing to do with the order structure of $H$ : if $V^{*} V=I$ but $V V^{*} \neq I$, then $\sigma(V)=\mathbf{D}$ necessarily holds. This follows from well-known facts about the structure of partial isometries, but holds in somewhat greater generality:

Proposition 3.5. Suppose that $\mathcal{A}$ is a complex Banach algebra with identity $I$ and that $V \in \mathcal{A}$ has a left inverse $U$. If there exists $\lambda \in \rho(V)$ such that $1 / \lambda \in \rho(U)$, then $V$ is invertible. In particular, if $\mathcal{A}$ is equipped with a conjugate-linear involutory anti-isomorphism $*$, and if $V^{*} V=I$ and $\mathrm{r}(V) \leq 1$, then either $V V^{*}=I$ or $\sigma(V)=\mathbf{D}$.

Proof. For $\lambda \in \rho(V)$, we have

$$
\begin{aligned}
& (I-\lambda U)\left[-V(\lambda I-V)^{-1}\right] \\
& \quad=-V(\lambda I-V)^{-1}+\lambda(\lambda I-V)^{-1} \\
& \quad=(\lambda I-V)(\lambda I-V)^{-1}=I .
\end{aligned}
$$

Hence if $(1 / \lambda) \in \rho(U)$ also, then

$$
\begin{aligned}
(I-\lambda U)^{-1} & =-V(\lambda I-V)^{-1}, \\
-(I-\lambda U)^{-1}(\lambda I-V) & =V,
\end{aligned}
$$

making $V$ the product of two invertible elements and hence invertible. If the setting is a $*$-algebra, $V^{*} V=I$, and $\mathrm{r}(V) \leq 1$, then $(\mathbf{C} \backslash \mathbf{D}) \subseteq \rho(V)$ by hypothesis, so $V$ can fail to be invertible if and only if $\sigma\left(V^{*}\right) \supseteq \mathbf{D}^{\circ}$; but $\sigma(V)=\operatorname{conj}\left(\sigma\left(V^{*}\right)\right)$, so $V$ fails to be invertible if and only if $\sigma(V)=\mathbf{D}$ - and of course $V$ is invertible if and only if $V V^{*}=I$.

Finally, we offer the following proof of a known theorem [4], [8], using approximation by polynomials with nonnegative coefficients. Our belief is that this proof is somewhat more transparent than previous arguments.

Theorem 3.6. Let $X$ be a real Banach space and $K \subseteq X$ a closed cone with $X=$ $\overline{K-K}$. If $A \in \mathcal{L}_{\mathbf{R}}(X)$ is an operator for which $A[K] \subseteq K$, and if its resolvent $R_{A}(\lambda)$ has a pole on the circle $\{\lambda:|\lambda|=\mathrm{r}(A)\}$, then $\mathrm{r}(A) \in \sigma(A)$.

Proof. With no loss of generality we can assume $\mathrm{r}(A)=1$. Let $\lambda_{1}$ be a pole of order $N$ of $R_{A}(\lambda)$ on the unit circle, and assume first that $\lambda_{1}$ is not real (i.e., $\lambda_{1} \neq-1$, since in the only other case we are finished); then $\bar{\lambda}_{1}$ is also a pole of the resolvent, with the principal parts at the two poles related as in (5.08) below. For the purpose of achieving a contradiction, assume $1 \notin \sigma(A)$. Let $\beta:=\sup \{\operatorname{Re}(\lambda): \lambda \in \sigma(A)\}<1$. Let $\sigma_{*}:=\left(\sigma(A) \backslash\left\{\lambda_{1}, \bar{\lambda}_{1}\right\}\right) \subseteq \mathbf{D}$, and let $\Sigma_{*} \subseteq \mathbf{D}$ be the convex hull of $\sigma_{*} \cup(\beta \cdot \mathbf{D})$. Since $1, \lambda_{1}$ and $\overline{\lambda_{1}}$ are extremal points of $\mathbf{D}$, they do not belong to $\Sigma_{*}$. Let 
$\delta:=\min \left\{\operatorname{dist}\left(\lambda, \Sigma_{*}\right): \lambda=1, \lambda_{1}, \bar{\lambda}_{1}\right\} ;$ then $\Sigma_{* *}:=\Sigma_{*}+(\delta / 2) \cdot \mathbf{D}$ is a closed, convex, symmetric set containing $\sigma_{*}$ in its interior but not containing $\lambda_{1}, \bar{\lambda}_{1}$, or 1 . Finally, let $\Sigma$ be the set formed by uniting $\Sigma_{* *}$ with the line segment having endpoints 0 and $2 \cdot \lambda_{1}$ and with the conjugate of that line segment. It is evident that $\Sigma$ and $\Sigma_{* *}$ are symmetric with respect to the real line and star-shaped with respect to the origin, and that neither contains 1 ; thus $\kappa=\sup \left(\Sigma \cap \mathbf{R}^{+}\right)<1$, indeed $\kappa \leq(1 / 2)(\beta+1)<1$.

Let $f \in \mathrm{S}(\Sigma)$ be a nonnegative real-valued function which is identically zero on $\Sigma_{* *}$ and strictly positive at $\lambda_{1}$ and $\bar{\lambda}_{1}, e . g ., f(z)=\operatorname{dist}\left(z, \Sigma_{* *}\right)$. If we take $V=\mathbf{C} \backslash \Sigma_{* *}$, then $V \subseteq \mathbf{C} \backslash((1 / 2)(\beta+1) \cdot \mathbf{D})$ and so a fortiori $V \subseteq \mathbf{C} \backslash(\kappa \cdot \mathbf{D})$; since $\Sigma_{* *}$ is a convex body, $V$ is connected. $(V \cap \Sigma)$ consists of a line segment with $\lambda_{1}$ in its interior and the conjugate of that line segment, so it has two-dimensional Lebesgue measure zero. Corollary 2.4 above thus implies that $f$ and $-f$ are uniform limits of sequences of polynomials with nonnegative coefficients, say $\left\{p_{n}(z)\right\}$ and $\left\{q_{n}(z)\right\}$ respectively. Let $L: \mathcal{C}(\Sigma) \rightarrow \mathcal{C}(\Sigma)$ be the operator of "complex integration along the line from 0 to $z$ ", i.e., $[L g](z)=\int_{0}^{z} g(\zeta) d \zeta$, where the integral is taken along the line segment joining those two points; evidently $L$ is linear and continuous in the uniform norm. If we set $P_{n}(z):=\left[L^{N-1} p_{n}\right](z)$, then these form a sequence of polynomials with nonnegative coefficients that converges to $L^{N-1} f$ uniformly on $\Sigma$, and the $k$-th derivatives converge uniformly to $L^{N-k-1} f$ for $k=0, \ldots, N-1$. The operators $P_{n}\left(A_{\mathbf{C}}\right)=P_{n}(A)_{\mathbf{C}}$ are real and send $K$ into $K$. By the holomorphic functional calculus, we see that

$$
\begin{aligned}
P_{n}\left(A_{\mathbf{C}}\right)=\sum_{\nu=0}^{N-1} \frac{P_{n}^{(\nu)}\left(\lambda_{1}\right)}{\nu !}\left(A_{\mathbf{C}}-\lambda_{1} I\right)^{\nu} E & +\sum_{\nu=0}^{N-1} \frac{P_{n}^{(\nu)}\left(\bar{\lambda}_{1}\right)}{\nu !}\left(A_{\mathbf{C}}-\bar{\lambda}_{1} I\right)^{\nu} J E J \\
& +\frac{1}{2 \pi i} \int_{\gamma_{0}} P_{n}(\lambda) R_{A}(\lambda) d \lambda
\end{aligned}
$$

where $E=\operatorname{Res}\left[\left.R_{A}(\lambda)\right|_{\lambda=\lambda_{1}}\right]$ and $\gamma_{0}$ is a contour in $\Sigma_{* *}$ winding once around $\sigma_{*}$. As $n \rightarrow \infty, P_{n}(\lambda) \rightarrow 0$ uniformly on $\gamma_{0}$, while for each $\nu=0, \ldots, N-1, P_{n}^{(\nu)}\left(\lambda_{1}\right) \rightarrow$ $\left[L^{N-\nu-1} f\right]\left(\lambda_{1}\right)$ and similarly at $\bar{\lambda}_{1}$. Passing to the limit in (3.05) thus produces an operator

$$
P:=\sum_{\nu=0}^{N-1} \frac{\left[L^{N-\nu-1} f\right]\left(\lambda_{1}\right)}{\nu !}\left(A_{\mathbf{C}}-\lambda_{1} I\right)^{\nu} E+\sum_{\nu=0}^{N-1} \frac{\left[L^{N-\nu-1} f\right]\left(\bar{\lambda}_{1}\right)}{\nu !}\left(A_{\mathbf{C}}-\bar{\lambda}_{1} I\right)^{\nu} J E J
$$

of the type of (5.09) below, which is nonzero (because the coefficient for $\nu=N-1$ is $f\left(\lambda_{1}\right) \neq 0$ ) and for which $P[K] \subseteq K$. On the other hand, exactly the same construction employing $\left\{q_{n}(z)\right\}$ instead of $\left\{p_{n}(z)\right\}$ would show that $-P[K] \subseteq K$; we have thus reached the contradiction that the nonzero operator $P$ must be the zero operator. Achieving the contradiction in the case where $\lambda_{1}=-1$ follows the same pattern, but with the simplifications produced by constructing operators of the form (5.10) rather than (5.09).

\section{A Counterexample, and Concluding Remarks}

Let $H$ be a real Hilbert space, $K \subseteq H$ be a closed, total cone in $H$, and $B$ : $H \rightarrow H$ be a normal operator for which $B[K] \subseteq K$. In $\S 3$ above we showed that if $\sigma(B)$ satisfied certain topological and measure-theoretic conditions, then $\operatorname{r}(B)$ was necessarily an element of $\sigma(B)$. One might reasonably suspect that those 
constraints on $\sigma(B)$ were artificial ones that simply made it possible for us to adapt the methods of Toland [12] to the treatment of normal operators, and one might conjecture that $\mathrm{r}(B) \in \sigma(B)$ necessarily holds whenever $B$ is a normal operator that maps a closed total cone into itself. In this section, however, we shall construct an example of a normal operator B that leaves a closed total cone invariant but for which $\mathrm{r}(B) \in \sigma(B)$ fails. The spectrum of the operator $B$ is a circle - so it separates some component of any neighborhood of each of its points from $\infty$ - and its existence indicates that when $B[K] \subseteq K$ but $K$ is not normal, then topological properties of the spectrum of $B$ play an intrinsic rôle in forcing $\mathrm{r}(B) \in \sigma(B)$, rather than being merely an artifact of the known proofs of various affirmative results.

Theorem 4.1. There exist a real separable Hilbert space $H$, a bounded normal linear operator $B: H \rightarrow H$, and a closed total cone $K \subseteq H$, such that $B[K] \subseteq K$ but $\mathrm{r}(B) \notin \sigma(B)$.

In fact, we shall construct $B$ in the form $B=-U-\alpha I$, where $U: H \rightarrow H$ is a unitary operator and $\alpha$ is a sufficiently small positive real number. The cone $K$ that we shall construct is inspired by an example of Bonsall's [4, ex. (iv), pp. 57-58], although our major difficulty, the proof that $K$ is total, has no analogue in [4].

Proof. We begin by establishing some fixed notation. In the proofs of Theorem 4.1 and Lemma $4.2, H$ will denote $\ell^{2}(\mathbf{Z}, \mathbf{R})$, the real Hilbert space of "doubly infinite sequences" $\left\{x_{j}\right\}_{j \in \mathbf{Z}}$ for which $\sum_{j \in \mathbf{Z}}\left|x_{j}\right|^{2}<\infty$. We shall consistently denote the right shift operator mapping $H$ onto itself by $U$. As usual, $e_{j}$ will denote the element of $H$ whose $j$-th coördinate is 1 and whose remaining coördinates are 0 ; thus $U$ is specified by saying that $U\left(e_{j}\right)=e_{j+1}$ for all $j \in \mathbf{Z}$.

Our first lemma is basically a restatement of well known facts, but for completeness we sketch a proof.

Lemma 4.2. If $U$ and $H$ are defined as above and if $\alpha$ is a positive real number, then (a): $\sigma(-U-\alpha I)=\{\zeta-\alpha:|\zeta|=1\}$, and (b): $\mathrm{r}(-U-\alpha I)=1+\alpha$.

Proof. When we complexify $H=\ell^{2}(\mathbf{Z}, \mathbf{R})$ as described in $\S 5$ below, we see that

$$
H_{\mathbf{C}}=\left\{\left(\begin{array}{l}
\left\{x_{j}: j \in \mathbf{Z}\right\} \\
\left\{y_{j}: j \in \mathbf{Z}\right\}
\end{array}\right):\left\{x_{j}: j \in \mathbf{Z}\right\},\left\{y_{j}: j \in \mathbf{Z}\right\} \in \ell^{2}(\mathbf{Z}, \mathbf{R})\right\} .
$$

From the definitions of the inner products in $H_{\mathbf{C}}$ and $\ell^{2}(\mathbf{Z}, \mathbf{C})$ respectively, it is clear that the 1-1 correspondence

$$
\left(\begin{array}{l}
\left\{x_{j}: j \in \mathbf{Z}\right\} \\
\left\{y_{j}: j \in \mathbf{Z}\right\}
\end{array}\right) \longleftrightarrow\left\{x_{j}+i \cdot y_{j}: j \in \mathbf{Z}\right\}
$$

between these two spaces is $\mathbf{C}$-linear and unitary (onto). Comparing the shift operators on these two spaces, one sees easily that the operator $U_{\mathbf{C}}=\left(\begin{array}{cc}U & 0 \\ 0 & U\end{array}\right)$ on $H_{\mathbf{C}}$ is unitarily equivalent to the right shift operator on $\ell^{2}(\mathbf{Z}, \mathbf{C})$ under that 11 correspondence. It is well known that the spectrum of the latter operator is $\{\lambda \in \mathbf{C}:|\lambda|=1\}$. For example, since $\|U\|=1$ and $\left\|U^{-1}\right\|=1$, the C. Neumann series $(\lambda I-U)^{-1}=\sum_{n=0}^{\infty} U^{n} / \lambda^{n+1}$ and its analogue for $U^{-1}$ show that $\sigma(U) \subseteq \mathbf{T}$. On the other hand, for any $N \in \mathbf{Z}, N \geq 0$, and any $0 \leq \theta \leq 2 \pi$ we may define an element $z^{(\theta, N)} \in \ell^{2}(\mathbf{Z}, \mathbf{C})$, of norm one, by setting

$$
z_{n}^{(\theta, N)}= \begin{cases}e^{i n \theta} / \sqrt{2 N+1}, & -N \leq n \leq N, \\ 0, & \text { otherwise }\end{cases}
$$


and it is routine to verify that $\left\|\left(e^{i \theta} I-U\right) z^{(\theta, N)}\right\|=\sqrt{2 /(2 N+1)}$, so that each $e^{i \theta} \in \mathbf{T}$ belongs to $\sigma(U)$. Once we know the spectrum of $U$, assertions (a) and (b) follow as trivial consequences of the spectral mapping theorem [14, p. 227].

Note that Lemma 4.2 implies that $1+\alpha=\mathrm{r}(-U-\alpha I) \notin \sigma(-U-\alpha I)$.

Proof of the theorem, continued. With $H$ and $U$ as before, fix an $\alpha$ with $0<\alpha<$ $1 / 3$ and define $B$ by

$$
B=-U-\alpha I
$$

The lemma implies that $\mathrm{r}(B)=1+\alpha \notin \sigma(B)$. It remains for us to construct a closed, total cone $K$ with $B[K] \subseteq K$. Fix a $\delta>0$ with $3 \alpha \leq 1-\delta$, and for each $z \in I_{\delta}=\{z \in \mathbf{R}:-1+(\delta / 2) \leq z \leq-1+\delta\}$, define $v^{z} \in H$ by

$$
v^{z}:=\sum_{k=0}^{\infty} z^{k} e_{k}
$$

With $\langle\cdot, \cdot\rangle$ denoting the inner product on $H$, define the set $K \subseteq H$ by

$$
K:=\left\{a \in H:\left\langle a,\left(B^{*}\right)^{j}\left(v^{z}\right)\right\rangle \geq 0 \text { for all } z \in I_{\delta} \text { and all } j \geq 0\right\} .
$$

Evidently if $a \in K$ then also $B(a) \in K$, because

$$
\left\langle B(a),\left(B^{*}\right)^{j}\left(v^{z}\right)\right\rangle=\left\langle a,\left(B^{*}\right)^{j+1}\left(v^{z}\right)\right\rangle \geq 0
$$

for all $j \geq 0$ and all $z \in I_{\delta}$. As the reader can easily check, the fact that $K$ is a closed wedge in $H$ follows immediately from its definition. It remains to be shown that $K \cap(-K)=\{0\}$ and - the harder part — that $K$ is total in $H$.

The identity

$$
\left(B^{*}\right)^{k}=(-1)^{k}\left(U^{*}+\alpha I\right)^{k}=(-1)^{k} \sum_{j=0}^{k}\left(\begin{array}{l}
k \\
j
\end{array}\right) \alpha^{k-j}\left(U^{*}\right)^{j}
$$

is an instance of the binomial theorem. For a fixed $z \in I_{\delta}$, this identity implies that with $v=v^{z}$, and using the notation

$$
\left(\left(B^{*}\right)^{k}(v)\right)_{m}:=\text { the } m \text {-th coördinate of }\left(B^{*}\right)^{k}(v),
$$

we have

$$
\left(\left(B^{*}\right)^{k}(v)\right)_{m}=(-1)^{k} \sum_{j=0}^{k}\left(\begin{array}{l}
k \\
j
\end{array}\right) \alpha^{k-j}\left(\left(U^{*}\right)^{j}(v)\right)_{m} .
$$

With $v_{m}$ denoting the $m$-th coördinate of $v$, so $v_{m}=z^{m}$ for $m \geq 0$ while $v_{m}=0$ for $m<0$, the relation (4.09) then tells us that

$$
\left(\left(B^{*}\right)^{k}(v)\right)_{m}=(-1)^{k} \sum_{j=0}^{k}\left(\begin{array}{c}
k \\
j
\end{array}\right) \alpha^{k-j} v_{m+j} .
$$

Classifying the three possible cases of (4.10), we see that

$$
\left(\left(B^{*}\right)^{k}(v)\right)_{m}= \begin{cases}(-1)^{k} \sum_{j=0}^{k}\left(\begin{array}{c}
k \\
j
\end{array}\right) \alpha^{k-j} z^{m+j}=(-1)^{k} z^{m}(z+\alpha)^{k}, & m \geq 0 \\
(-1)^{k} z^{m} \sum_{j=|m|}^{k}\left(\begin{array}{c}
k \\
j
\end{array}\right) \alpha^{k-j} z^{j}, & -k \leq m<0 \\
0, & m<-k\end{cases}
$$


If $a$ is an element of $H$, we deduce from (4.06) and (4.11) that $a \in K$ if and only if the inequalities

$$
(-1)^{k}(z+\alpha)^{k} \sum_{m=0}^{\infty} a_{m} z^{m}+(-1)^{k} \sum_{m=-k}^{-1} a_{m} z^{m}\left(\sum_{j=|m|}^{k}\left(\begin{array}{c}
k \\
j
\end{array}\right) \alpha^{k-j} z^{j}\right) \geq 0
$$

hold for all $k \geq 0$ and all $z \in I_{\delta}$ (where of course $a_{m}$ denotes the $m$-th coördinate of $a$ ).

In order to show that $K$ is a (proper) cone, we must show that if $a \in K \cap(-K)$ then $a=0$, i.e., that all the coördinates $a_{m}$ are zero. Because $a \in K \cap(-K)$, equality must hold in (4.12) for all $k \geq 0$ and all $z \in I_{\delta}$. These equalities tell us immediately that $a_{m}=0$ for all indices $m \geq 0$, because for $k=0$ the equalities are

$$
\sum_{m=0}^{\infty} a_{m} z^{m}=0 \quad \text { for all } z \in I_{\delta}
$$

and so $a_{m}=0$ for all $m \geq 0$ by the identity theorem for holomorphic functions (the function defined by the sum $\sum a_{m} z^{m}$ is easily seen to be holomorphic on $\mathbf{D}$ and indeed to belong to $H^{2}(\mathbf{D})$ ). For coördinates with negative index, we may reason by induction. Suppose we have shown that $a_{m}=0$ for all $m>-N$, where $N$ is a positive integer; then taking $k=N$ in (4.12), we see that there is only one nonzero term on the left-hand side, and it gives us $(-1)^{N} a_{-N}=0$, i.e., $a_{-N}=0$. This completes the induction and shows us that if $a \in K \cap(-K)$, then $a_{m}=0$ for all $m \in \mathbf{Z}$, so $a=0$ as we wished.

To see that $K-K$ is a dense subspace of $H$, we begin by considering the subspace and subcone defined by

$$
H_{+}:=\left\{a \in H: a_{m}=0 \text { for all } m \leq 0\right\} \quad \text { and } \quad K_{+}:=K \cap H_{+} .
$$

Since $(-1)^{k}(z+\alpha)^{k}>0$ for all $k \geq 0$ and $z \in I_{\delta}$, we see as a consequence of (4.12) that for an element $a \in H_{+}$the condition

$$
\sum_{m=1}^{\infty} a_{m} z^{m} \geq 0 \quad \text { for all } z \in I_{\delta}
$$

is necessary and sufficient for $a$ to belong to $K_{+}$. In particular, since $z<0$ for all $z \in I_{\delta}$, if $a$ is known to belong to $H_{+}$, then for $a \in K_{+}$to hold it is sufficient that $(-1)^{m} a_{m} \geq 0$ for all $m \geq 1$. The trivial decomposition

$$
\left\{a_{m}: m \in \mathbf{Z}\right\}=\sum\left\{a_{m} e_{m}:(-1)^{m} a_{m} \geq 0\right\}-\sum\left\{a_{m} e_{m}:(-1)^{m} a_{m}<0\right\}
$$

of an element $a \in H_{+}$thus represents it as an element of $K_{+}-K_{+}$.

Next, for each positive integer $N$ define a subspace $H_{N}$ and a subcone $K_{N}$ by

$H_{N}:=\left\{a \in H: a_{m}=0\right.$ for all $m>0$ and all $\left.m<-N\right\} \quad$ and $\quad K_{N}:=K \cap H_{N}$.

Evidently $H_{N}$ is a real Hilbert space of finite dimension $N+1$ and therefore isometrically isomorphic to $\mathbf{R}^{N+1}$, and because $K$ is a cone, $K_{N}$ is a (proper) cone in $H_{N}$. In order to show that $H_{N}=K_{N}-K_{N}$, it will consequently suffice to show that $K_{N}$ has nonempty interior in $H_{N}$. If we can establish this, therefore, we shall know that $\left(H_{N}+H_{+}\right) \subseteq K-K$ for every positive integer $N$. Since $N$ was arbitrary, this will tell us precisely that if $a$ is an element of $H$ for which there exists an $N$ such that $a_{m}=0$ for all $m<-N$, then $a \in K-K$, and since those $a$ 's form a 
dense subspace of $H$ we shall have proved that $K-K$ is total and established the validity of our example.

Thus all that remains to prove is that $K_{N}$ has nonempty interior in $H_{N}$. To that end it suffices to exhibit a number $R>0$ such that if $a=\left\{a_{m}\right\} \in H_{N}$ satisfies $a_{0} \geq R$ and $\left|a_{m}\right| \leq 1$ for $-N \leq m \leq-1$, then $a \in K_{N}$. For $a \in H_{N}$, the relations (4.12) imply that $a \in K_{N}$ if and only if for all $z \in I_{\delta}$ and all $k \geq 0$ the inequalities

$$
(-1)^{k}(z+\alpha)^{k} a_{0}+(-1)^{k}\left[\sum_{m=-\min (N, k)}^{-1} a_{m} z^{m}\left(\sum_{j=|m|}^{k}\left(\begin{array}{c}
k \\
j
\end{array}\right) \alpha^{k-j} z^{j}\right)\right] \geq 0
$$

hold. If we assume that $a_{0} \geq R>0$ and that $\left|a_{m}\right| \leq 1$ for $-N \leq m<0$, and we recall that $(-1)^{k}(z+\alpha)^{k}>0$ for all $z \in I_{\delta}$ and observe that

$$
\sum_{j=|m|}^{k}\left(\begin{array}{l}
k \\
j
\end{array}\right) \alpha^{k-j} z^{m}=(z+\alpha)^{k}-\sum_{j=0}^{|m|-1}\left(\begin{array}{l}
k \\
j
\end{array}\right) \alpha^{k-j} z^{j}
$$

then we see that for the relations (4.18) to hold it is sufficient that the relations

$$
R \geq \sum_{m=-\min (N, k)}^{-1}|z|^{m} \cdot\left(\frac{1}{|z+\alpha|^{k}}\right) \cdot\left|(z+\alpha)^{k}-\sum_{j=0}^{|m|-1}\left(\begin{array}{c}
k \\
j
\end{array}\right) \alpha^{k-j} z^{j}\right|
$$

hold for all $k \geq 0$ and all $z \in I_{\delta}$. To estimate the right-hand sides of (4.20), observe that

$$
\begin{aligned}
& \frac{1}{|z+\alpha|^{k}} \cdot\left|(z+\alpha)^{k}-\sum_{j=0}^{|m|-1}\left(\begin{array}{c}
k \\
j
\end{array}\right) \alpha^{k-j} z^{j}\right| \leq 1+\sum_{j=0}^{|m|-1}\left(\begin{array}{c}
k \\
j
\end{array}\right)\left(\frac{\alpha}{|z+\alpha|}\right)^{k}\left(\frac{|z|}{\alpha}\right)^{j} \\
& \leq 1+\left(\frac{\alpha}{|z+\alpha|}\right)^{k} \cdot\left(\frac{|z|}{\alpha}\right)^{|m|-1} \cdot\left(\sum_{j=0}^{|m|-1}\left(\begin{array}{c}
k \\
j
\end{array}\right)\right) .
\end{aligned}
$$

Our choice of $\delta$ and $\alpha$ insures that for all $z \in I_{\delta}$ and $k \geq 0$ we have

$$
\left(\frac{\alpha}{|z+\alpha|}\right)^{k} \leq\left(\frac{1}{2}\right)^{k}
$$

It is trivial that

$$
\sum_{j=0}^{|m|-1}\left(\begin{array}{l}
k \\
j
\end{array}\right) \leq \sum_{j=0}^{k}\left(\begin{array}{l}
k \\
j
\end{array}\right)=2^{k}
$$

Combining the relations (4.21)-(4.23), we see that the relations (4.20) will hold if $R$ can be so chosen that for all $z \in I_{\delta}$ and $k \geq 0$ we have

$$
R \geq \sum_{m=-\min (N, k)}^{-1}|z|^{m} \cdot\left[1+\left(\frac{|z|}{\alpha}\right)^{|m|-1}\right] .
$$

It is easy to check that (4.14) will be satisfied if

$$
R \geq N \cdot\left(\frac{1}{1-\delta}\right)^{N} \cdot\left[1+\left(\frac{1}{\alpha}\right)^{N-1}\right],
$$

and that completes the proof of the theorem. 
Remark 4.3. If $B$ is the operator constructed in Theorem 4.1 above and if $f(z)$ is a function holomorphic on a neighborhood of $\sigma(B)$ and satisfying $f(\bar{z})=\overline{f(z)}$ for all $z$ in its domain, then $f(B)$ is normal. For many choices of $f(z)$, one will also have $f(B)[K] \subseteq K$. This is particularly easy to see when $f(z)$ is a polynomial in $z$ with nonnegative coefficients, or when $f(z)=(\lambda-z)^{-1}$, where $1-\alpha<\lambda \in \mathbf{R}$. In this way one obtains a source of further counterexamples to a priori plausible extensions of the results given here. For example, if $f(z)=2 \alpha z+z^{2}+\epsilon z^{3}$, where $\epsilon>0$, and if one sets $B_{1}=f(B)$, then one sees easily that $B_{1}$ is normal (and real) and that $B_{1}[K] \subseteq K$. However, if $\epsilon$ is sufficiently small then one can show that $\mathrm{r}\left(B_{1}\right) \notin \sigma\left(B_{1}\right)$ and that - in contrast with the case of the operator $B$ produced by the detailed construction that is Theorem 4.1 - there exists no real $\lambda \in \sigma\left(B_{1}\right)$ for which $|\lambda|=\mathrm{r}\left(B_{1}\right)$. We omit the detailed verification of this statement.

Remark 4.4. The cone $K$ constructed in the proof of Theorem 4.1 above is not normal.

This is not difficult to check. To see it, let $N \geq 2$ be an even integer and suppose that $c \in H$ is a sequence for which the $j$-th coördinate $c_{j}$ of $c$ equals zero unless $j=N$ or $j=2 N$. If $c_{N}>0$, it follows from the relations (4.12) that $c \in K$ if and only if

$$
\left(1+\left(\frac{c_{2 N}}{c_{N}}\right) z^{N}\right) \geq 0 \text { for all } z \in I_{\delta} .
$$

If $a \in H$ is defined by setting $a_{j}=0$ for $j \neq N$ or $2 N, a_{N}=1$ and $a_{2 N}=$ $[1-(\delta / 2)]^{-N}$, and $b \in H$ is defined by setting $b_{j}=0$ for $j \neq N$ or $2 N, b_{N}=2$ and $b_{2 N}=1$, then one can use the relations (4.26) to check that $a, b$ and $b-a$ are elements of $K$. However, $\|b\|=\sqrt{5}$ for all even integers $N \geq 2$, while $\|a\| \rightarrow \infty$ as $N \rightarrow \infty$, so $K$ cannot be normal.

Remark 4.5. For the operator $B$ constructed in the proof of Theorem 4.1, one can see that $(-1-\alpha) \in \sigma(B)$ but that $-1-\alpha$ does not belong to the point spectrum of $B$. The following proposition, which is a special case of a result of [7], shows that this fact is not accidental.

Proposition 4.6. Let $K$ be a closed, total cone in a reflexive real Banach space $X$ and $B: X \rightarrow X$ be a bounded linear mapping for which $B[K] \subseteq K$. Suppose that the spectral radius $r=\mathrm{r}(B)$ is positive and that there exists a constant $M$ for which

$$
\left\|B^{n}\right\| \leq M r^{n} \text { for all } n \geq 1 \text {. }
$$

If there exist a complex $\lambda$ in the point spectrum of $B_{\mathbf{C}}$ and an integer $m \geq 1$ for which $\lambda^{m}=r^{m}$, then $r$ belongs to the point spectrum of $B$.

If $K$ is a closed, total cone in a real Hilbert space $H$ and $B: H \rightarrow H$ is a normal operator with $B[K] \subseteq K$, then the relations (4.27) are satisfied with $M=1$. If $r=\mathrm{r}(B)=\|B\|>0$ also holds, and there exist some $\lambda$ in the point spectrum of $B$ and some $m \geq 1$ such that $\lambda^{m}=r^{m}$, then Prop. 4.6 implies that $r$ must be an element of the point spectrum of $B$. In particular, if $-r \in \sigma(B)$ and $r \notin \sigma(B)$, then $-r$ cannot belong to the point spectrum of $B$.

Remark 4.7. Suppose $X$ is a real Banach space, $K \subseteq X$ is a closed, total cone and $U \in \mathcal{L}(X)$ is such that $\|U\|=1$ and $(-U)[K] \subseteq K$. Define $\alpha_{0}$ by

$$
\alpha_{0}:=\sup \{\alpha \geq 0:(-U-\alpha I)[K] \subseteq K\} .
$$


For the unitary operator $U$ of Theorem 4.1 above, we were able to find cones $K$ for which $\alpha_{0} \geq \beta$ held for any $\beta \leq 1 / 3$. We observe, however, that in all such cases $\alpha_{0} \leq 1$. This follows from the following simple observation, applied to $-U$.

Remark 4.8. Let $X$ be a real Banach space and $K \subseteq X$ be a closed wedge that is proper, i.e., in which there exists $u \in K$ for which $-u \notin K$. Let $V \in \mathcal{L}(X)$ be a linear operator for which $V[K] \subseteq K$. If $\alpha \in \mathbf{R}$ is such that $(V-\alpha I)[K] \subseteq K$, then $\alpha \leq \mathrm{r}(V)$.

Indeed, suppose there were such an $\alpha>\mathrm{r}(V)$. Then the $\mathrm{C}$. Neumann series $(\alpha I-V)^{-1}=\sum_{n=0}^{\infty} V^{n} / \alpha^{n+1}$ shows that $(\alpha I-V)^{-1}[K] \subseteq K$, but this fact combined with $(V-\alpha I)[K] \subseteq K$ would give

$$
-K=(-I)[K]=(V-\alpha I)(\alpha I-V)^{-1}[K] \subseteq K,
$$

and $K$ would not be proper.

\section{Remarks on Spectral Theory in Complexifications}

Let $X$ be a real Banach (or Hilbert) space. The standard way to do spectral theory on $X$ is to tensor $X$ with $\mathbf{C}$ over $\mathbf{R}$, setting $X_{\mathbf{C}}=\mathbf{C} \otimes_{\mathbf{R}} X$ and using the obvious multiplication. Since $\operatorname{dim}(\mathbf{C}: \mathbf{R})=2$, this comes to the same thing as forming

$$
X_{\mathbf{C}}=X \oplus_{\mathbf{R}} X=\left\{\left(\begin{array}{l}
x \\
y
\end{array}\right): x, y \in X\right\}
$$

and defining

$$
(\alpha+i \beta)\left(\begin{array}{l}
x \\
y
\end{array}\right)=\left(\begin{array}{c}
\alpha x-\beta y \\
\beta x+\alpha y
\end{array}\right)
$$

$X_{\mathbf{C}}$ is then a complex vector space and normable real TVS in an essentially unique way, and if for example one sets

$$
\left\|\left(\begin{array}{l}
x \\
y
\end{array}\right)\right\|^{2}=\frac{1}{2 \pi} \int_{-\pi}^{\pi}\left[\|(\cos t) x-(\sin t) y\|^{2}+\|(\sin t) x+(\cos t) y\|^{2}\right] d t,
$$

then it is routine to verify that this is a real norm that also satisfies $\left\|\lambda\left(\begin{array}{l}x \\ y\end{array}\right)\right\|=$ $|\lambda| \cdot\left\|\left(\begin{array}{l}x \\ y\end{array}\right)\right\|$ for complex $\lambda$. If the space $X$ was a Hilbert space with inner product $\langle\cdot, \cdot\rangle$, then setting

$$
\left\langle\left(\begin{array}{l}
x \\
y
\end{array}\right),\left(\begin{array}{l}
u \\
v
\end{array}\right)\right\rangle=\{[\langle x, u\rangle+\langle y, v\rangle]+i \cdot[-\langle x, v\rangle+\langle y, u\rangle]\}
$$

makes $X_{\mathbf{C}}$ a complex Hilbert space whose inner product norm is the same as the norm defined by (5.03).

In all cases it is instructive to think of $\mathcal{L}_{\mathbf{R}}\left(X_{\mathbf{C}}\right)$ as being given by matrices $\left(\begin{array}{ll}A & B \\ C & D\end{array}\right)$ with entries in $\mathcal{L}_{\mathbf{R}}(X)$, acting on $X_{\mathbf{C}}$ in the obvious way. Then the elements of $\mathcal{L}_{\mathbf{R}}\left(X_{\mathbf{C}}\right)$ that are $\mathbf{C}$-linear are just those that commute with multiplication by $i=$ $\left(\begin{array}{cc}0 & -I \\ I & 0\end{array}\right)$, i.e., matrices of the form $\left(\begin{array}{cc}A & -B \\ B & A\end{array}\right)$. A rather laborious but straightforward computation shows that if $X$ was a real Hilbert space, then the adjoint of $\left(\begin{array}{cc}A & -B \\ B & A\end{array}\right)$ on $X_{\mathbf{C}}$ is $\left(\begin{array}{ccc}A^{*} & B^{*} \\ -B^{*} & A^{*}\end{array}\right)$. A useful but non-C-linear-indeed, a conjugate-linear-map in $\mathcal{L}_{\mathbf{R}}\left(X_{\mathbf{C}}\right)$ is $J=\left(\begin{array}{cc}I & 0 \\ 0 & -I\end{array}\right)$, which sends $\left(\begin{array}{l}x \\ y\end{array}\right) \rightarrow\left(\begin{array}{c}x \\ -y\end{array}\right)$. (This can be viewed as $\tau \otimes I$ on $\mathbf{C} \otimes_{\mathbf{R}} X$, where $\tau: \lambda \rightarrow \bar{\lambda}$ is complex conjugation.) Clearly $J$ is an isometry and $J^{2}=I$. If $T \in \mathcal{L}_{\mathbf{C}}\left(X_{\mathbf{C}}\right)$ then $J T J$ is again $\mathbf{C}$-linear, and since $T \rightarrow J T J$ is a 
(real) inner automorphism of $\mathcal{L}_{\mathbf{R}}\left(X_{\mathbf{C}}\right)$ it preserves all the real-algebra operations of $\mathcal{L}_{\mathbf{C}}\left(X_{\mathbf{C}}\right)$; however, $T \rightarrow J T J$ is only $\mathbf{C}$ - conjugate-linear, and $J\left(\begin{array}{cc}A & -B \\ B & A\end{array}\right) J=\left(\begin{array}{cc}A & B \\ -B & A\end{array}\right)$, so $J$ is "complex conjugation" on $\mathcal{L}_{\mathbf{C}}\left(X_{\mathbf{C}}\right)$ also. It preserves the $*$ operation if $X$ is a Hilbert space. We shall call $J T J$ the conjugate of $T$ and thus distinguish it from the Hilbert-space adjoint. It is easily verified that the operations of taking conjugates and taking adjoints commute.

There is a natural isomorphism of $\mathcal{L}_{\mathbf{R}}(X)$ into $\mathcal{L}_{\mathbf{C}}\left(X_{\mathbf{C}}\right)$ given by

$$
A \rightarrow 1 \otimes_{\mathbf{R}} A \leftrightarrow\left(\begin{array}{cc}
A & 0 \\
0 & A
\end{array}\right) \stackrel{\text { def }}{=} A_{\mathbf{C}}
$$

Elements of the image are characterized by both being complex-linear and commuting with $J$ (i.e., satisfying $J T J=T$ ). The simple matrix computation that we gave above shows this, because $J T J=T$ holds if and only if $B=-B$. We shall call such elements of $\mathcal{L}_{\mathbf{C}}\left(X_{\mathbf{C}}\right)$ real. A real operator $T \in \mathcal{L}_{\mathbf{C}}\left(X_{\mathbf{C}}\right)$ can thus be identified with a unique operator belonging to $\mathcal{L}_{\mathbf{R}}(X)$; we have tried to call attention to such an identification whenever we made it.

Since $T \rightarrow J T J$ is a real automorphism of $\mathcal{L}_{\mathbf{C}}\left(X_{\mathbf{C}}\right)$, an operator $T$ has a left (or right, or two-sided) inverse if and only if $J T J$ does. By conjugate linearity we have

$$
(\lambda I-T) R_{T}(\lambda)=I \Longleftrightarrow(\bar{\lambda} I-J T J)\left(J R_{T}(\lambda) J\right)=I
$$

with a similar relation on the other side, so

$$
\lambda \in \rho(T) \Longleftrightarrow \bar{\lambda} \in \rho(J T J)
$$

or equivalently

$$
\lambda \in \sigma(T) \Longleftrightarrow \bar{\lambda} \in \sigma(J T J) .
$$

If $\lambda_{1} \in \sigma(T)$ is an isolated point of $\sigma(T)$, then locally

$$
R_{T}(\lambda)=\sum_{n=0}^{\infty} \frac{\left(T-\lambda_{1} I\right)^{n}}{\left(\lambda-\lambda_{1}\right)^{n+1}} E+(\text { analytic function of } \lambda),
$$

where $E=\operatorname{Res}\left[\left.R_{T}(\lambda)\right|_{\lambda=\lambda_{1}}\right]$ [14, Ch. VIII, §7, p. 225 ff.], and thus

$$
R_{J T J}(\zeta)=\sum_{n=0}^{\infty} \frac{\left(J T J-\bar{\lambda}_{1} I\right)^{n}}{\left(\zeta-\bar{\lambda}_{1}\right)^{n+1}} J E J+(\text { analytic function of } \zeta)
$$

(think of $\zeta$ as $\bar{\lambda}$ ) near $\bar{\lambda}_{1}$. The cases of this pair of equations that interest us here are those in which $T$ is real, so that $T=J T J-$ suppose $T=A_{\mathbf{C}}$. The spectrum of $A \in \mathcal{L}_{\mathbf{R}}(X)$ is then defined to be $\sigma\left(A_{\mathbf{C}}\right)$, so in these cases $\sigma(A) \subseteq \mathbf{C}$ is automatically symmetric with respect to $\mathbf{R}$ (a well-known two-dimensional example shows that while $\sigma(A)$ must be nonempty, it need not contain points of $\mathbf{R}$ ). If $\lambda_{1} \in \mathbf{R}$, then by uniqueness of Laurent series we have $E=J E J$; the residue, which is the spectral projection onto the spectral subspace of $X_{\mathbf{C}}$ belonging to $\left\{\lambda_{1}\right\}$, must be real and therefore identifiable with a (unique) idempotent in $\mathcal{L}_{\mathbf{R}}(X)$. If $\lambda_{1} \notin \mathbf{R}$, then $J E J$ is the spectral projection onto the spectral subspace belonging to $\left\{\bar{\lambda}_{1}\right\}$, and by the holomorphic functional calculus [14] we have $E \cdot(J E J)=(J E J) \cdot E=0$. It follows that $E+J E J$ is the projection onto the spectral subspace belonging to $\left\{\lambda_{1}, \bar{\lambda}_{1}\right\}$, and that this projection is real, so that, again, it can be identified with an idempotent in $\mathcal{L}_{\mathbf{R}}(X)$. If $\lambda_{1}$ is a pole of $R_{T}(\lambda)$ of order $N$ - this occurs 
when and only when the operator $T-\lambda_{1} I$ is nilpotent of order $N$ on the spectral subspace belonging to $\lambda_{1}$, so in particular $\lambda_{1}$ is an eigenvalue of $T$ - then the same properties hold for the operator $J T J$ and the eigenvalue $\bar{\lambda}_{1}$. It is now easy to verify that in the case where $T=A_{\mathbf{C}}$ and $\lambda_{1} \in \sigma(A) \backslash \mathbf{R}$ is a pole of order $N$, the restriction of $A$ to the subspace $(E+J E J)[X]$ satisfies a (minimal) real polynomial equation of degree exactly $2 N$. Indeed, if $p(\lambda)$ is a real polynomial for which $p(A) \mid(E+J E J)[X]=0$, then $p(A)$ is a complex polynomial for which both $p\left(A_{\mathbf{C}}\right) \mid E\left[X_{\mathbf{C}}\right]=0$ and $p\left(A_{\mathbf{C}}\right) \mid J E J\left[X_{\mathbf{C}}\right]=0$, and since those two subspaces are $A_{\mathbf{C}}$-direct summands in $X_{\mathbf{C}}$, both of the prime powers $\left(\lambda-\lambda_{1}\right)^{N}$ and $\left(\lambda-\bar{\lambda}_{1}\right)^{N}$ must divide $p(\lambda)$ in $\mathbf{C}[\lambda]$, so the real polynomial $\left[\left(\lambda-\lambda_{1}\right)\left(\lambda-\bar{\lambda}_{1}\right)\right]^{N}$ must divide $p(\lambda)$ in $\mathbf{R}[\lambda]$. Now of course $\left[\left(A_{\mathbf{C}}-\lambda_{1} I\right)\left(A_{\mathbf{C}}-\bar{\lambda}_{1} I\right)\right]^{N} \mid(E+J E J)\left[X_{\mathbf{C}}\right]=0$, whence the same statement is true without the subscript "C"s - and so the prime power $\left(\lambda^{2}-2 \operatorname{Re}\left[\lambda_{1}\right] \lambda+\left|\lambda_{1}\right|^{2}\right)^{N} \in \mathbf{R}[\lambda]$ must be the minimal polynomial of $A \mid(E+J E J)[X]$. One sees in an analogous manner that any operator of the form

$$
\sum_{n=0}^{N-1} \alpha_{n}\left(A_{\mathbf{C}}-\lambda_{1} I\right)^{n} E+\sum_{n=0}^{N-1} \bar{\alpha}_{n}\left(A_{\mathbf{C}}-\bar{\lambda}_{1} I\right)^{n} J E J
$$

will be real-hence well-defined as an element of $\mathcal{L}_{\mathbf{R}}(X)$ - and will be the zero operator if and only if all the (complex) coefficients $\left\{\alpha_{n}\right\}_{n=0}^{N-1}$ equal zero. The corresponding assertions in the case where $\lambda_{1} \in \mathbf{R}$ is a pole of order $N$ are even simpler to verify, since the operators

$$
\sum_{n=0}^{N-1} \alpha_{n}\left(A_{\mathbf{C}}-\lambda_{1} I\right)^{n} E=\left[\sum_{n=0}^{N-1} \alpha_{n}\left(A-\lambda_{1} I\right)^{n} E\right]_{\mathbf{C}}
$$

(where the coefficients $\left\{\alpha_{n}\right\}_{n=0}^{N-1}$ must now be real) are already real and the degree of the minimal polynomial of $A \mid E[X]$ must be $N$.

\section{REFERENCES}

1. R. Askey, Orthogonal Polynomials and Special Functions, Regional Conference Series in Applied Math., SIAM, Philadelphia, 1975. MR 58:11288

2. L. Bieberbach, Lehrbuch der Funktionentheorie Vol. II, Teubner, Leipzig, 1931. MR 40:2823b (later ed.)

3. E. Bishop, A minimal boundary for function algebras, Pacific J. Math. 9 (1959), 629-642. MR 22:191

4. F. F. Bonsall, Linear operators in complete positive cones, Proc. London Math. Soc. (3) 8 (1958), 53-75. MR 19:1183c

5. L.-K. Hua, Harmonic analysis of functions of several complex variables in the classical domains, Translation from the Russian, Amer. Math. Soc., Providence, R. I., 1963. MR 30:2162

6. S. Kantorovitz, Classification of operators by means of their operational calculus, Transactions Amer. Math. Soc. 115 (1965), 194-224. MR 33:7855

7. R. D. Nussbaum, Eigenvectors of order-preserving linear operators, [accepted by] J. London Math. Soc..

8. H. H. Schaefer, Halbgeordnete lokalkonvexe Vektorräume. II, Math. Ann. 138 (1959), 259-286. MR 21:5135

9. H. H. Schaefer, Topological Vector Spaces, Macmillan, New York, 1966. MR 33:1689

10. L. Schwartz, Théorie des Distributions, Hermann \& Cie.; Tome I, 1957; Tome II, 1959. MR 21:6534; MR 22:8322

11. E. C. Titchmarsh, The Theory of Functions, 2nd ed., Oxford Univ. Press, 1939 (corr. printing 1968). 
12. J. F. Toland, Self-adjoint operators and cones, J. London Math. Soc. (2) 53 (1996), 167-183. MR 96k: 47040

13. D. V. Widder, The Laplace Transform, Princeton Univ. Press, 1941. MR 3:232d

14. K. Yosida, Functional Analysis, 6th ed., Springer-Verlag, Berlin, 1980. MR 82i:46002

Department of Mathematics, Rutgers University, New Brunswick, New Jersey 089032101

E-mail address: nussbaum@math.rutgers.edu

E-mail address: bwalsh@math.rutgers.edu 